\title{
Data-driven computational models reveal perceptual simulation in word processing
}

\author{
Marco A. Petillia,b,*, Fritz Güntherc, Alessandra Vergallito ${ }^{\mathrm{a}, \mathrm{b}}$, Marco Ciapparelli ${ }^{\mathrm{a}}$, \\ Marco Marellia,b \\ ${ }^{a}$ Psychology, Università degli Studi di Milano-Bicocca, Milan, Italy \\ ${ }^{b}$ NeuroMI - Milan Center for Neuroscience, Milan, Italy \\ ${ }^{c}$ Department of Psychology, University of Tübingen, Tübingen, Germany
}

\begin{abstract}
Note: This is the author's preprint version of the article (date: 13. 11. 2020). The final article is accepted for publication in Journal of Memory and Language.
\end{abstract}

In their strongest formulation, theories of grounded cognition claim that concepts are made up of sensorimotor information. Following such equivalence, perceptual properties of objects should consistently influence processing, even in purely linguistic tasks, where perceptual information is neither solicited nor required. Previous studies have tested this prediction in semantic priming tasks, but they have not observed perceptual influences on participants' performances. However, those findings suffer from critical shortcomings, which may have prevented potential visually grounded/perceptual effects from being detected. Here, we investigate this topic by applying an innovative method expected to increase the sensitivity in detecting such perceptual effects. Specifically, we adopt an objective, data-driven, computational approach to independently quantify vision-based and language-based similarities for prime-target pairs on a continuous scale. We test whether these measures predict behavioural performance in a semantic priming mega-study with various experimental settings. Vision-based similarity is found to facilitate performance, but a dissociation between vision-based and language-based effects was also observed. Thus, in line with theories of grounded cognition, perceptual properties can facilitate word processing even in purely linguistic tasks, but the behavioural dissociation at the same time challenges strong claims of sensorimotor and conceptual equivalence.

Keywords: perceptual simulation; semantic priming; distributional semantic models; convolutional neural networks; grounded cognition.

This work was supported by a Research Fellowship (no. 392225719) from the German Research Foundation (DFG), awarded to Fritz Günther, and by grant 2017-1633 from the Fondazione Cariplo-Regione Lombardia, awarded to Marco Marelli. All item materials, data, and analysis scripts are available at https://osf.io/n7gqa/.

* Corresponding author. E-mail address: marco.petilli@unimib.it 
According to proposals in the field of grounded cognition, language comprehension involves the reactivation of the sensorimotor (i.e., perceptual and motor) states acquired during everyday experience (Barsalou, 2008; Fischer, 2012; Zwaan \& Madden, 2005). In this perspective, sensorimotor processing would play a crucial role as a constitutive part of the meaning-making stream. In a discussion of this approach, Mahon (2015b) identifies that in the strongest formulation of grounded cognition, "conceptual content is represented entirely in terms of sensorimotor information and computations over sensorimotor content. Stated differently, modalityspecific information (plus computations over that content) is necessary and sufficient to support all conceptual processing” (Mahon, 2015b, p. 423).

In line with this hypothesis, converging neuroimaging and neuropsychological evidence suggests that conceptual and sensorimotor processing share the same neural and cognitive mechanisms. First, several neuroimaging studies have described a somatotopically organized activation of sensorimotor areas during conceptual processing (e.g., Hauk et al., 2004). Furthermore, neuropsychological studies have reported conceptual deficits after sensory-motor impairments, suggesting a contribution of sensorimotor information in semantic processing (e.g., Trumpp et al., 2013). However, although neuroimaging and neuropsychological studies suggest an important role for the sensorimotor systems in language processing, they do not demonstrate a clear equivalence between the two systems. In fact, simultaneous activation of the sensorimotor and conceptual systems, as described in neuroimaging studies, could reflect the spreading activation between two functionally independent but connected systems (see Mahon, 2015a). Moreover, other clinical studies critically challenge a strong grounded perspective equating conceptual with sensorimotor processing by reporting cases of sensorimotor deficit without conceptual impairment (see Binder \& Desai, 2011; Mahon \& Caramazza, 2005).

\section{Perceptual effects in word processing}

Behavioural studies have further contributed to this research line by investigating the degree of association between sensorimotor and semantic processing in linguistic tasks (see, for example, Glenberg \& Kaschak, 2002; Lachmair et al., 2011; Zwaan et al., 2002). In fact, if conceptual and sensorimotor processing are equivalent, one would expect that perceptual properties of objects should consistently influence conceptual processing even in purely language-based semantic tasks. In order to investigate this prediction, several studies have tested the impact of visual aspects of word-denoted objects in semantic priming paradigms, where visual processing is neither solicited nor required (Flores D'Arcais et al., 1985; Pecher et al., 1998; Schreuder et al., 1984). Typically, in semantic priming paradigms, responses (naming or lexical decision) to a target word (e.g., cherry) are faster when the target is preceded by linguistically related/similar (e.g., banana), relative to unrelated/dissimilar (e.g., table), prime words (the semantic priming effect; Hutchison, 2003; Meyer \& Schvaneveldt, 1971). Following grounded-cognition predictions, if sensorimotor processing is (or critically contributes to) conceptual processing, the same facilitation will be expected for visually related/similar (e.g., ball-cherry) vis-à-vis visually unrelated/dissimilar (e.g., table-cherry) prime-target pairs.

Interestingly, the first studies investigating visionbased similarity effects in semantic priming (Flores D'Arcais et al., 1985; Schreuder et al., 1984) had supported, at least partially, the grounded perspective, even before this theoretical proposal was fully formulated. These studies have employed a factorial design in which pairs of words rated as linguistically related, visually related, or both linguistically and visually related were presented as stimuli in a series of priming experiments. The authors found vision-based similarity effects (i.e., an advantage for word pairs denoting objects with similar visual properties, such as ball-cherry vis-à-vis table-cherry) in both a lexical decision (LDT) and a naming task (NT), with a stimulus onset asynchrony (SOA) of $400 \mathrm{~ms}$. Conversely, language-based effects (i.e., an advantage for linguistically related pairs such as banana-cherry vs. table-cherry) have been found only in the LDT, but not in the NT responses (in line with the literature indicating generally weaker priming effects in the NT as compared to the LDT; Lucas, 2000). However, these early studies have raised criticisms concerning 
the experimental procedure and employed materials (Pecher et al., 1998). First, from a procedural point of view, the prime remained on the screen during the presentation of the target. This aspect may have promoted strategies that overly draw attention to the relation between the prime and target (Shelton \& Martin, 1992). Second, concerning the employed material, Pecher et al. (1998) have criticized the adoption of different word sets in unrelated and related conditions. This would have introduced a confounding between the experimental conditions and the employed materials. Third, some stimuli have been repeated as prime, as target, or as both prime and target. This may have promoted the use of strategies affecting responses and, therefore, the observed priming effects (Shelton \& Martin, 1992; for further details, see Pecher et al., 1998).

Pecher et al. (1998) have addressed these issues in six different priming experiments (LDT and NT, all with an SOA of $350 \mathrm{~ms}$ ). In order to avoid the problematic aspects of previous studies (Flores D'Arcais et al., 1985; Schreuder et al., 1984), they introduced several adjustments: the target was presented after the prime and not simultaneously to it; word pairs rated as linguistically and/or visually related were selected and presented in order to make each word appear only once during the experiment; each relatedness condition was compared to an unrelated control condition (i.e., unrelated word pairs constructed by recombining prime-target pairs within the set), thus ensuring that any difference between the two conditions was due to the prime-target relation. With these modifications, the experiments did not find evidence in favor of the alleged influence of sensory information in word processing: while Pecher at al. (1998) consistently observed language-based priming effects, vision-based effects emerged only when the priming task was preceded by perceptual activation tasks, which strongly encouraged strategic retrieval of the perceptual properties of the word-denoted objects. Thus, these results suggest that visual properties of the denoted object do not automatically contribute to word processing (see also Lebois et al., 2015; Ostarek \& Huettig, 2017, 2019). In a subsequent meta-analysis based on the studies mentioned above, Hutchison (2003) concluded that there is no reliable automatic visual priming effect in word-processing behavioural tasks. A more recent study (Yee et al., 2012) has further corroborated this conclusion, suggesting that conceptual processing in semantic priming is not automatically influenced by the colour of objects (i.e., no advantage for pairs such as emerald-cucumber) since facilitatory effects were traceable only when the semantic priming task was preceded by a task enforcing visual processing.

\section{Methodological and theoretical concerns with respect to previous studies}

From the evidence described so far, behavioural studies on semantic priming seem not to be in line with the neuroimaging and neuropsychological findings we discussed earlier. However, these behavioural investigations suffer from critical shortcomings, which may have prevented potential vision-based effects from being detected. Let us first consider methodological issues. Earlier studies typically have characterized vision-based similarity in dichotomic terms, rated as either "related" or "unrelated". This traditional convention to depend on extreme cases of visually or linguistically related or unrelated word pairs, while ignoring the vast majority of items lying in between these two poles, has directly limited the range of investigation of these effects to very small datasets, usually including a few dozen items (see Pecher et al., 1998). On the one hand, this aspect limits the generalization of the obtained results. On the other hand, both the reliance on small datasets and the dichotomization of independent variables are associated with limitations in terms of statistical power (see Keuleers \& Balota, 2015).

In addition to these methodological considerations, other problematic aspects are related to the use of human behaviour as both dependent and independent variables. First, the studies described so far have defined the category of the employed stimuli by relying on human intuitions, i.e., by asking participants to rate to what extent two words are perceptually or semantically similar. However, such judgments of similarity are strongly biased by individual beliefs, feelings, and knowledge about the objects to be judged (see, for example, Simmons \& Estes, 2008). Furthermore, to be objective and accurate, human judgments of similarity should, ideally, be based on the whole set of commonalities and dissimilarities in the multiple dimensions characterizing two object representations. However, 
human limitation imposes each rater to select and rely only on a limited set of object properties to provide a similarity judgment. This is further exacerbated by the fact that the properties that people take into account in a similarity judgment and the weight they attribute to each property are not necessarily fixed across similarity judgments for different object pairs (see Tversky, 1977). As an example (based on James, 1984/2001), people may rely heavily on the brightness when they provide a judgment of vision-based similarity between two bright concepts (e.g., "moon" and "lamp"). Differently, they may tend to give more importance to the shape when they judge the visionbased similarity between two round objects (e.g., "moon" and "ball"). All these aspects affect people's assessment of similarity in a way that is difficult to control, is far from being objective, and creates noise in the estimations of similarity. This becomes particularly problematic when these data are used as independent variables that are supposed to accurately measure the object properties under investigation. This makes clear the need for more objective measures of similarity that systematically take into account the same, ideally as exhaustive as possible, set of object properties and compare them in a way that is independent of individual beliefs, feelings, and knowledge in general.

In addition to these considerations, there is also a fundamental theoretical concern with the methodology adopted in previous studies, where estimates of visionbased and language-based similarity were built on human intuitions (either researcher judgments or participant ratings). Within this approach, one aims at explaining one type of behavioural phenomenon (priming effects, the explanandum; Hempel \& Oppenheim, 1948) by relying on another type of behavioural phenomenon (similarity judgments, which is used as the explanans) (for detailed versions of this argument, see Jones et al., 2015; Westbury, 2016). However, this just leaves the explanation at the same level of description as the thing to be explained, without actually tackling the cognitive phenomenon of interest: Where do these similarity judgments come from in the first place? Do we really assume that priming effects occur because participants explicitly judge the items to be more or less similar? Or do we rather infer that a third factor - such as our experience with the world and the stimuli in it-gives rise to associations between concepts (see Bhatia, 2017), which then result in similarity judgments on the one hand and priming effects on the other hand? We argue that the second option is more plausible: the perceived similarity between two entities is a function of their properties and the way our system processes them. This perceived similarity is what can influence behaviour, for example, similarity judgments and priming effects. However, adopting this perspective requires us to dramatically shift the level of explanation away from the outcome level of directly observable psychological states towards the input level that gives rise to these psychological states (see Westbury, 2016). In other words, we need to (a) adequately describe stimulus properties (in this case, vision-based and language-based properties of the primes and the target) by relying on independent sources approximating the speaker's experience and (b) formulate an explicit model about how the cognitive system processes these properties.

In addition to these epistemological considerations, starting from (an approximation of) actual experience also has important practical implications: rating studies are by nature restricted to the stimuli that were presented to participants; in contrast, an experiencebased model straightforwardly generates predictions for any number of stimuli outside the original set, because it explicitly formalizes how stimulus properties translate to the level of the observed phenomenon. All these considerations make clear the need for more objective approaches and independent sources from which vision-based and language-based similarity can be estimated.

\section{Data-driven modelling, a solution}

In the present study, we address these theoretical and methodological issues by applying an innovative method for investigating perceptual priming. Specifically, we investigate vision-based similarity effects in the largest semantic-priming database available (Hutchison et al., 2013), employing fully implemented and data-driven state-of-the-art computational models borrowed from artificial intelligence, and in particular Natural Language Processing (Mikolov et al., 2013) and Computer Vision (Chatfield et al., 2014). These models allow us to characterize the degree of both language-based and vision-based similarity as the continuous variables 
they are, derived directly from independent, first-order sources of data (natural language samples and collections of images) (Keuleers \& Marelli, 2020). This type of computational operationalization has long been called for in the literature on grounded cognition (Andrews et al., 2014; Pezzulo et al., 2011, 2013), but until recently (Bruni et al., 2014; Lazaridou et al., 2017), an adequate cognitively oriented computational framework has been missing.

In reference to our methodological and theoretical concerns, previous studies in the lexical-semantic domain have already demonstrated that this computational approach even outperforms estimates based on human intuition on empirical grounds: Mandera et al. (2017) have found that semantic priming effects are better explained by language-based similarity measures obtained from data-driven models rather than by measures based on human ratings. Extending upon this previous research, we employ an approach that enables us to derive, in a comparable way, both estimates of language-based similarity (through computational models trained on text corpora) and estimates of vision-based similarity (through computational models trained on image databases) for prime-target pairs in order to thoroughly investigate effects of perceptual similarity in word processing.

Based on these data-driven continuous measures of similarity, we tested the grounded cognition hypothesis postulating perceptual simulation in semantic processing (e.g., Barsalou, 2008). Specifically, we compared the effects of languagebased and vision-based similarity in behavioural data released through the Semantic Priming Project (SPP; Hutchison et al., 2013). If perceptual simulation of word-denoted concepts is routinely involved in lexical processing, one would expect that even in such a purely linguistic task, the vision-based similarity between prime and target will predict priming effects over and above what is predicted by their languagebased similarity. This potential outcome would strongly suggest a contribution of sensorimotor information (here, in the form of visual simulation) in the conceptual processing stream.

Note, however, that the strongest formulation of grounded cognition, assuming a functional equivalence between language processing and sensorimotor activation (Glenberg, 2015), would predict language-based and sensorimotor-based effects to be consistently associated across different linguistic tasks. Semantic priming data from the SPP are ideally suited to investigate these claims: the SPP, including responses for two different paradigms (NT vs. LDT) and stimulus-onset asynchronies (short-SOA $250 \mathrm{~ms}$ vs. long-SOA $1200 \mathrm{~ms}$ SOA), allows to test whether language-based and vision-based effects consistently co-occur across different tasks and over the time course of processing. Indeed, establishing cross-task robustness is currently considered one of the main challenges in grounded cognition (Ostarek \& Huettig, 2019). If we observe vision-based similarity effects in at least one condition, this would indicate that perceptual information is automatically activated even when the task at hand does not require it. On the other hand, a potential dissociation between visionbased and language-based effects in these semantic priming conditions would challenge the strong grounded perspective equating conceptual with sensorimotor processing (Glenberg, 2015).

\section{Computational Framework and Measures}

\section{Language-based similarity}

Language-based (or text-based) similarity estimates were obtained via a distributional semantics framework. Distributional semantic models approximate word meanings through an analysis of distributions of lexical co-occurrences and have been successfully applied in cognitive science as models of semantic memory (see Günther et al., 2019; Jones \& Mewhort, 2007; Landauer \& Dumais, 1997). In particular, we collected text-based similarity estimates using the SNAUT web interface provided by Mandera et al. (2017). The estimates were based on a 300dimension distributional model constructed using the Continuous Bag of Words (CBOW) algorithm of the word2vec toolkit (Mikolov et al., 2013), which estimates numerical vector representations for word meanings through a neural network architecture predicting a target word given its context. Context was defined as a 6-word window around the target word. The distributional model was constructed by running the algorithm on the concatenation of a 385-millionword OpenSubtitles corpus (www.opensubtitles.org) and the 2 billion words of the UK Web as Corpus (UKWaC), a corpus obtained through web crawling 
(http://wacky.sslmit.unibo.it; Ferraresi, Zanchetta, Baroni, \& Bernardini, 2008). Text-based similarity between two word-items was then defined in terms of the cosine between their corresponding vectors1.

Note that distributional semantic models are based on the distributional hypothesis that words that are similar in meaning occur in similar contexts (Harris, 1954), implying that a word meaning arises (or to the very least can be approximated) from the context in which it is used (Lenci, 2008). Contextual information, hence, has a decisive impact on the training of such text-based models. It is also worth noting that such a computational system can find its cognitive underpinnings in learning theories of similarity-based generalization in language (Anderson \& Milson, 1989; Rescorla \& Wagner, 1972; see Landauer \& Dumais, 1997; Mandera et al., 2017; Hollis, 2017, for detailed arguments) as well as within grounded-framework approaches that centrally rely on the notion of co-occurrence between and within units of linguistic and sensorimotor experience as a meaning-forming mechanism (Zwaan \& Madden, 2005; see Günther et al., 2019).

\section{Vision-based similarity}

Vision-based (or image-based) similarity estimates between word-pairs were obtained by adopting stateof-the-art methods from image-recognition systems for computer vision (Chatfield et al., 2014; Krizhevsky et al., 2012). For each word-item, a set of pictures associated with its referent (ranging from 100 to 200, depending on the number of images available) was retrieved from ImageNet, a large-scale database of labelled images (Deng et al., 2009), adopting the hierarchical category structure of WordNet (Miller, 1998). For words associated with more than one category in ImageNet, we selected the category including the largest number of images.

As our image-recognition system, we employed MatConvNet (Vedaldi \& Lenc, 2015), which allowed us to extract vector representations of the visual

\footnotetext{
${ }^{1}$ Cosine similarity measures the angle between two n-dimensional vectors, and determines whether two vectors are pointing in the same direction within the n-dimensional space (Han et al., 2012). It ranges from -1 , meaning two vectors point in exactly the opposite direction, to 1 , meaning two vectors point in exactly the same direction. It takes a value of 0 when two vectors are orthogonal. A systematic evaluation study by Bullinaria \& Levy (2007) has
}

features included in each picture: first, all the pictures selected from ImageNet were processed through the pre-trained VGG-F Convolutional Neural Network (CNN) (Chatfield et al., 2014). This model is set up as an eight-layer CNN model trained at predicting a label on the basis of a picture (for a more detailed description of the model architecture, see Vedaldi \& Lenc, 2015). Essentially, this type of model learns to extract visual features that are consistently denoted through a given word; for example, the model can learn the visual representation of a table by individuating the typical pattern of visual features found in a large number of images labelled "table". The cognitive plausibility of visual representations obtained from this type of models has been validated in previous studies, which have shown that simulations based on estimates from CNNs are in line with explicit intuitions of human participants (Bracci et al., 2019; Lazaridou et al., 2017). Following previous literature (e.g., Lazaridou et al., 2017), we extracted, for each image, the 4096-dimensional vector representation in the second-to-last layer (fc7), which has been argued to capture complex, abstract, gestalt-level representations of objects (LeCun et al., 2015; Smith et al., 2017; Zeiler \& Fergus, 2014). For each word stimulus, a unique vision-based representation was then estimated as its prototypical visual activation, operationalized as the average vector for all pictures extracted for the given word (Günther, Petilli, \& Marelli, 2020; Günther, Petilli, Vergallito, et al., 2020; Lazaridou et al., 2017). Items that deviated too far (in terms of cosine similarity) from the mean activation value within a category (interquartile ranges over 1.5) were not included in the averaging process. Image-based similarity between item pairs was finally computed as the cosine similarity between their respective vector representations (Figure 1).

identified cosine similarity as the most reliable similarity metric, which is indeed used for both linguistic (Baroni et al., 2014; Mandera et al., 2017) and visual models (Bruni et al., 2014; Lazaridou et al., 2017; Zhang et al., 2018). 


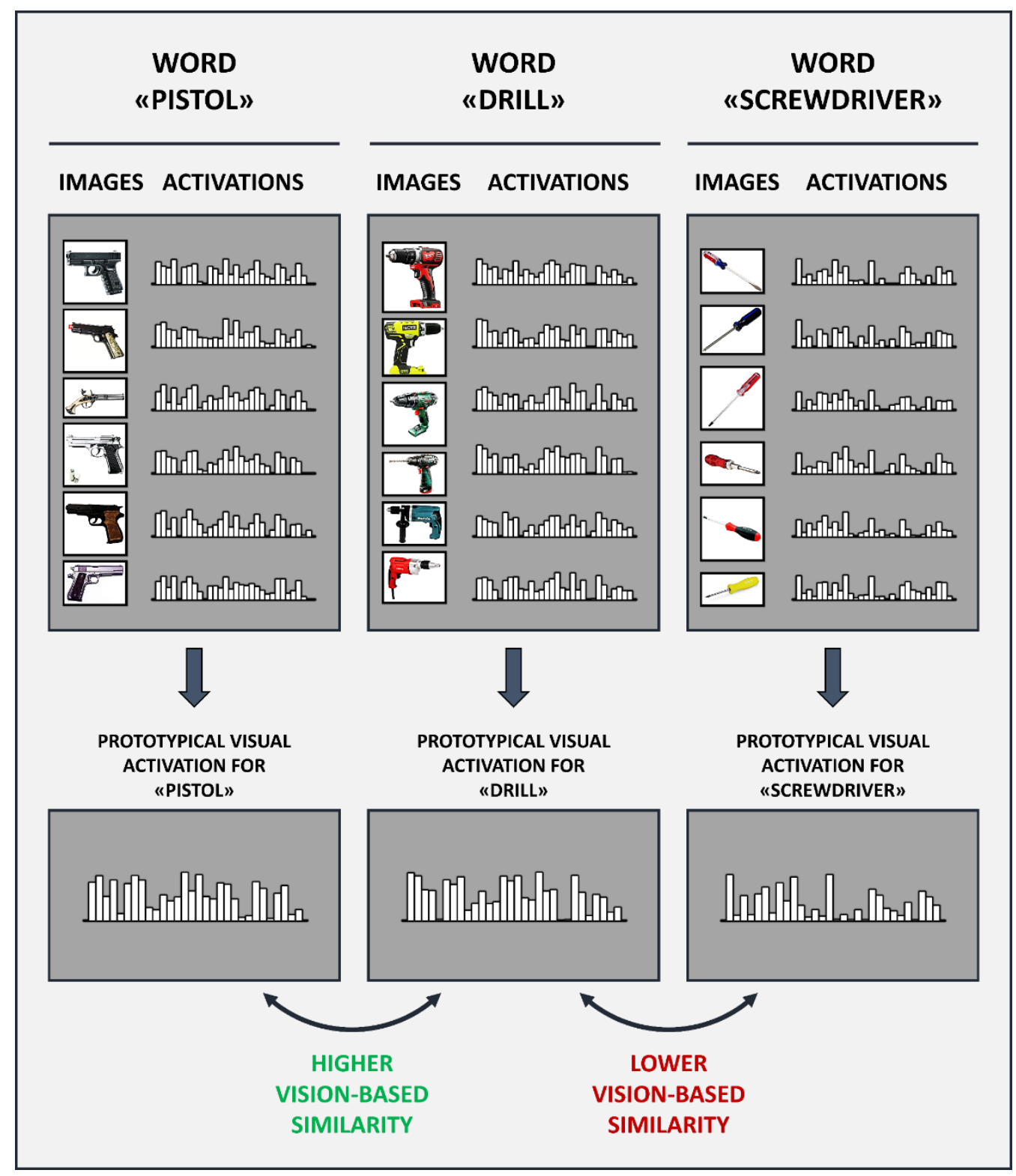

Fig 1: Schematic representation of vision-based estimation of visual similarity for three sample words: pistol, drill and, screwdriver. For each word, the prototypical visual activation was computed as the average activation in the second-to-last layer of the Convolutional Neural Network (CNN) across 200 images associated with the word. Vision-based similarity between two items was then estimated as the cosine between their respective prototypical visual activation. 


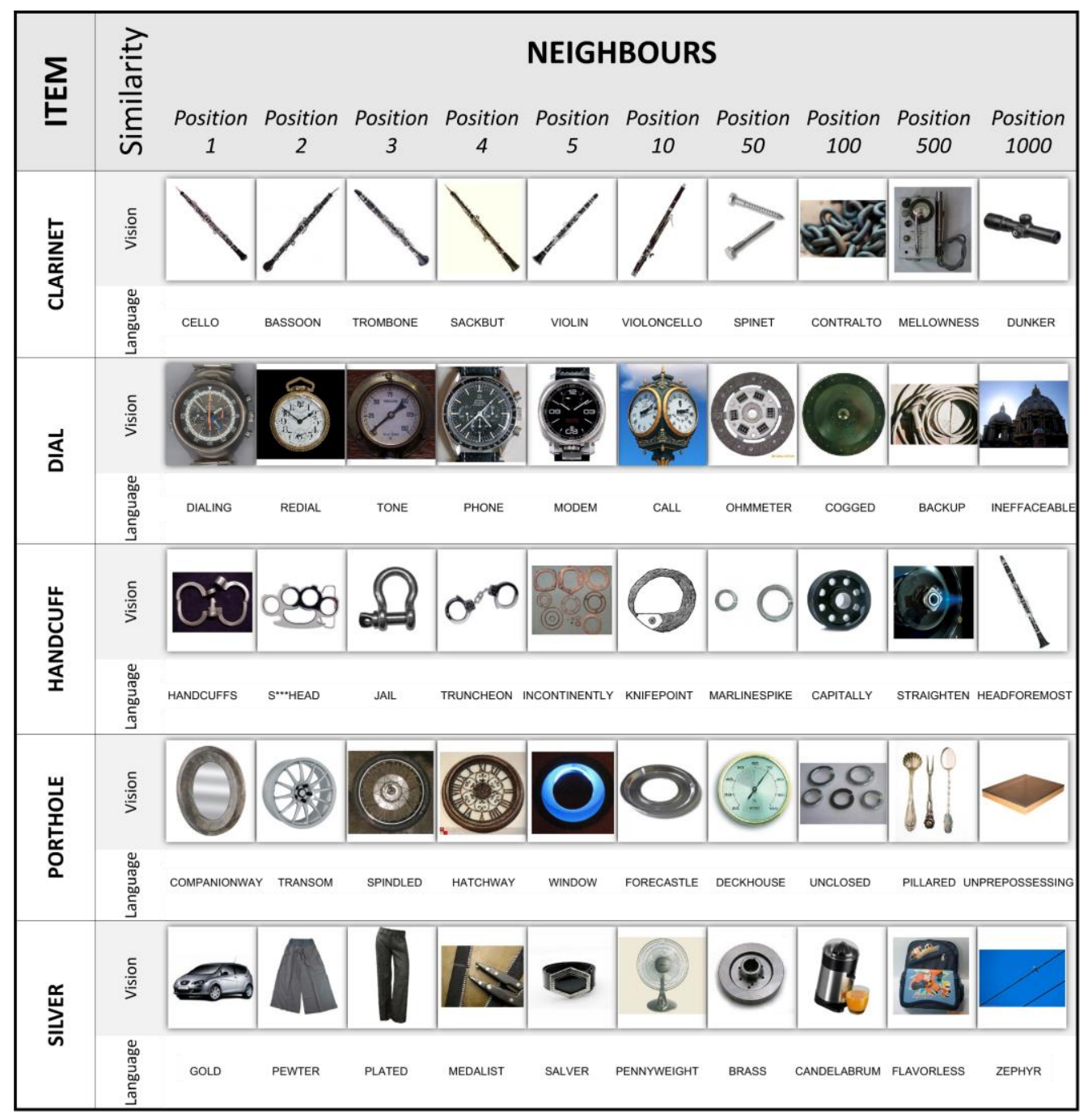

Fig 2: The figure reports, for five target word-items (left column), a list of vision-based neighbours (i.e., visual items sorted according to their vision-based similarity with the target) and language-based neighbours (i.e., linguistic items sorted according to their language-based similarity with the target), as predicted by the employed distributional models. Vision-based and language-based similarities decrease from left to right. Each visual neighbor is represented through the image whose visual vector is closest to the prototype vector of the corresponding ImageNet category (see method section).

Figure 2 provides a qualitative intuition of the representations captured by this model, as well as its dissociation with the predictions of the text-based approach discussed in the previous section. For a few example words, Figure 2 reports automatically extracted neighbours obtained through the text-based vis-à-vis the image-based system. Whereas the imagebased model builds on properties related to the shape and the colour of the object, the text-based model captures relations that are better described in lexicalsemantic terms.

Note that the method applied here to extract visual prototypes is expected to approximate rich visual experience associated with a given object. In fact, the CNN model was also fed with images of objects represented in their diverse daily contexts. As a result, the representations induced by the model are influenced not only by the visual features of the object 
itself but also by the visual contexts in which the object appears. These different aspects are represented in the prototype vector proportionally to the extent they contribute to the perceptual experience of the object. Importantly, this approach, in the definition of the image data used to train the model, is consistent with the idea of situated simulation proposed in the grounded cognition literature (Barsalou et al., 2008). According to this view, perceptual experience is never context-independent, that is, objects are never perceived alone against an empty background in our everyday interactions with the world. Information on the visual context is itself part of the perceptual experience of the object, which speaks for the psychological plausibility of the visual representations computed in the present study.

\section{Validation of the computational systems}

A large number of empirical studies have established the validity of distributional semantic models as approximations of the human semantic memory (e.g., Günther, Dudschig, \& Kaup, 2016; Jones, Kintsch, \& Mewhort, 2006; Landauer \& Dumais, 1997). Extensive testing has demonstrated that this approach is reliable in predicting explicit word-meaning intuitions and measures concerning semantic processing, such as similarity ratings, categorization, synonym tests, or free associations (Baroni et al., 2014; see Günther et al., 2019 for an overview). Notably, estimates from distributional models have also been validated against several semantic-priming results, including the dataset adopted here (Hutchison et al., 2013). Specifically, the study by Mandera et al. (2017) has shown that the textbased similarity between primes and targets of the SPP reliably predicts semantic facilitations in both lexical decision and word naming and that the model adopted in the present study provides well-performing textbased estimates in predicting SPP behavioural data (see also Wingfield \& Connell, 2019). Critically, Mandera et al. (2017) have demonstrated that measures based on distributional semantics outperform traditional measures obtained from human participants (i.e., human-based word-associations and feature-norms) in explaining SPP behavioural variance in both LDT and NT. This finding empirically corroborates the arguments in favour of the computational approaches presented in the Introduction.

To elaborate on Mandera et al.'s (2017) conclusions, we performed a follow-up analysis on the subsets of items employed in the present study (note that we could not employ all the item pairs of the SPP, but only those for which visual representations could be derived for both prime and target, as described in the next section). Using a regression approach, we compared text-based similarity estimates with SPP human-based relatedness classifications (related vs. unrelated) in predicting behavioural data across the different SPP conditions. Results showed that textbased similarity estimates consistently outperform human-based classifications. In LDT Short-SOA, $\Delta \mathrm{AIC}=-5.6$ indicates that text-based similarity estimates are 16.1 times more likely to have produced the behavioural data (in terms of Akaike Weight Ratio), and thus the normalized probability of preferring text-based estimates over human-based classifications as a model for the data is $\mathrm{p}=.94$ (Wagenmakers \& Farrell, 2004). In LDT Long-SOA, we obtained $\triangle \mathrm{AIC}=-2.1$, with an Akaike Weight Ratio of 2.9, and a normalized probability of preferring text-based over human-based estimates of $p=.74$. In NT Long-SOA, we obtained $\triangle \mathrm{AIC}=-4.7$, with an Akaike Weight Ratio of 10.4, and a normalized probability of preferring text-based over human-based estimates of $\mathrm{p}=.91$. A different pattern was only found for the NT short-SOA condition, where neither of the variables had significant impact on behavioural data (human-based predictor: $\mathrm{t}(1109)=1.796, \mathrm{p}=.073$; text-based estimates: $\mathrm{t}(1109)=-1.508, \mathrm{p}=.132)$. These results confirm that text-based similarity estimates are excellent predictors of semantic priming and hence represent a strong baseline against which potential perceptual effects can be tested.

How about image-related predictions from the CNN model - are they valid approximations of visionbased similarities? This issue has recently been addressed in a comprehensive study by Zhang, Isola, Efros, Shechtman, and Wang (2018). They collected ratings of perceptual similarity for a large set of images and showed that measures based on the representations induced by $\mathrm{CNN}$ models are very much in line with those obtained from human judgments (up to the point where Zhang et al. refer to them as "unreasonably effective perceptual similarity 
metrics" in the title of their paper). This result has been observed across a wide range of different architectures, including those adopted in the present study, indicating that our image-based estimates are ideally suited as approximations of vision-based similarity between objects.

In order to further establish the validity of our image-based estimates and their viability for testing perceptual priming, we tested the model predictions in a simulation study based on an item set previously employed in the perceptual-priming literature (Pecher et al., 1998). We compared, across 1000 permutations, model predictions for 29 visually related item pairs (e.g., orange-ball, pistol-drill,...) obtained from Pecher et al. (1998), and 29 visually unrelated item pairs (e.g., orange-coffee, pistol-ball, ...) obtained by randomly pairing the same items. Mean cosine similarity data obtained from each permutation were transformed into z-scores. The average cosine similarity for the visually related pairs was higher than the average cosine similarity for the random pairs in each of the 1000 permutations (Figure 3 ) and was 4.93 standard deviations higher than the average cosine similarity of the whole distribution (note that this analysis corresponds to a permutation test of significance with $\mathrm{p}<.001)$. This result supports the reliability of the model in capturing vision-based similarities and the viability of the obtained measures for investigating perceptual priming.

Our analyses, in conjunction with previous findings from the literature, indicate that the text-based and image-based models adopted here can respectively capture language-based and vision-based similarity between word-denoted items. Further evidence indicates that such models capture pieces of information that are largely independent from one another. The most impressive result in this regard comes from an fMRI study employing a word-reading task (i.e., a purely language-based task) by Anderson, Bruni, Lopopolo, Poesio, \& Baroni (2015), which showed that text-based and image-based vectors pattern with activations in brain areas that are segregated from each other. Specifically, they were able to discriminate activation related to image-based vectors in ventral-temporal and lateral-occipital object-selective areas, from activation related to text-
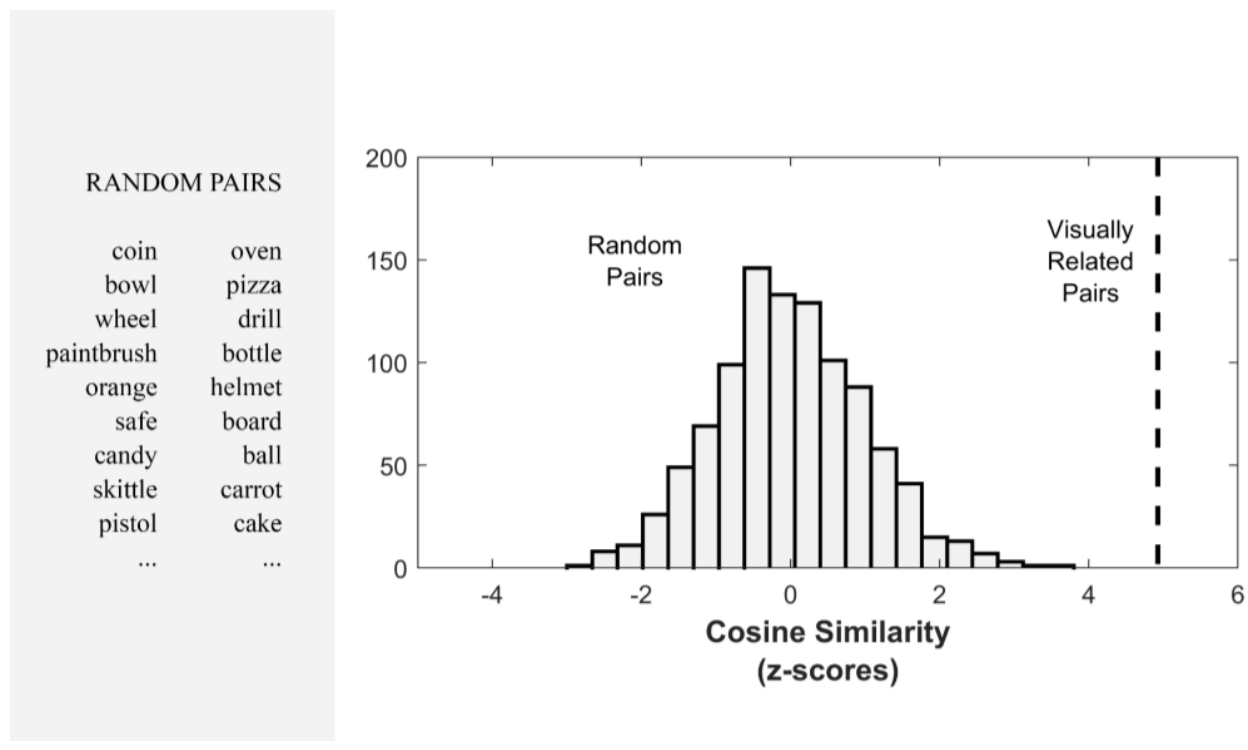

RELATED PAIRS

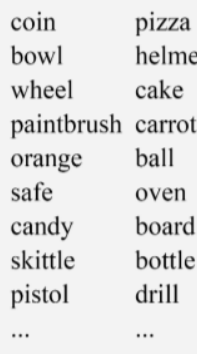

Fig 3: The dashed vertical line represents the average image-based cosine similarity computed for 29 item pairs rated as visually related. The histogram represents the distribution of average image-based cosine similarity for 1000 permutations of the same set of items in random pairings. Cosine similarity is expressed in z-scores. On the sides of the figure, there are some examples of random and related prime and target pairs (see Pecher et al., 1998). 
based vectors in posterior-parietal/lateraltemporal/inferior-frontal modality-independent areas. To the purpose of this study, this represents a crucial result: (i) it supports the validity of such image-based models in capturing perceptual effects in word processing, and (ii) it indicates that text-based and image-based representations capture independent properties, associated with dissociated processes at the neural level.

In order to provide further support to these conclusions at a behavioural level, we tested the validity of our model-based similarity measures in predicting human judgment for both vision-based and language-based similarity for 179 word-picture pairs obtained from De Groot, Koelewijn, Huettig, and Olivers, (2016). To this end, adopting a regression approach, we first evaluated the effects of text-based similarity estimates in predicting ratings of languagebased similarity (baseline model). Results showed that text-based estimates significantly predict ratings of language-based similarity $(\mathrm{t}(177)=12.93 ; \mathrm{p}<.001)$. In a second step, we added the image-based similarity estimates to the regression model and assessed whether this additional parameter improved the model fit. No improvement in goodness-of-fit was observed $(\mathrm{F}(1,176)=.244 ; \mathrm{p}=.622 ; \mathrm{r}$-squared change $=.0007)$, suggesting that image-based similarity estimates do not account for additional variance in language-based similarity judgments with respect to the one already explained by text-based estimates. Likewise, results from this model showed that only text-based estimates significantly predict ratings of language-based similarity $(\mathrm{t}(176)=12.186 ; \mathrm{p}<.001)$, while the effect of image-based similarity was not found to be significant $(\mathrm{t}(176)=.494 ; \mathrm{p}=.622)$ (Figure 4). The same analysis was then performed by considering human ratings of vision-based similarity on the same word-picture pairs (De Groot et al., 2016). Accordingly, we first evaluated the effects of imagebased similarity estimates in predicting ratings of vision-based similarity (baseline model). Results showed that image-based estimates significantly predict ratings of vision-based similarity $(\mathrm{t}$ (177) = 3.148; $\mathrm{p}=.002)$. After having established a baseline, we added to the regression model the text-based similarity estimates and assessed whether this additional parameter improved the model fit. No improvement in goodness-of-fit was observed (F (1,

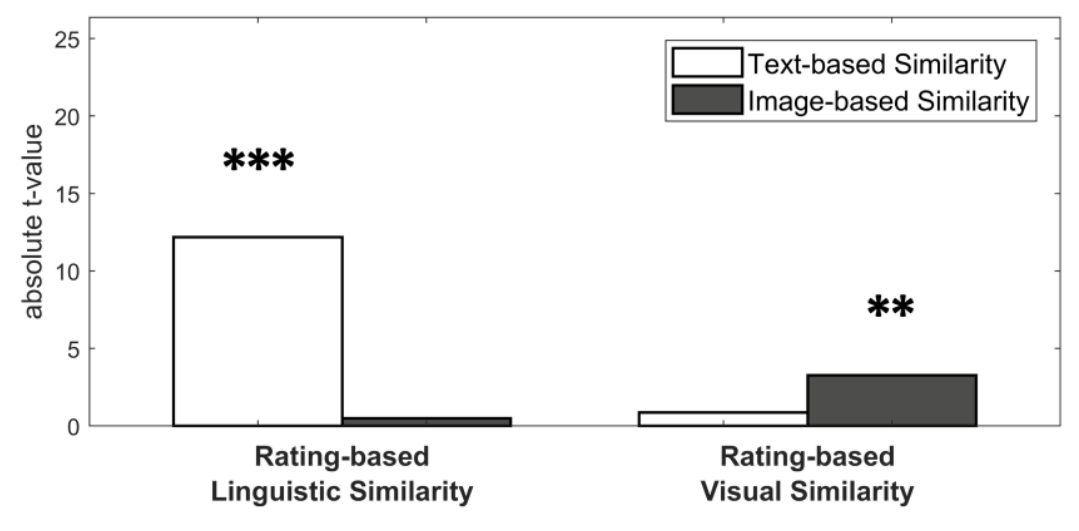

Note:* $\mathrm{p}<.05 ; * * \mathrm{p}<.01 ; * * * \mathrm{p}<.001$

Fig 4: Strength of the effects (i.e., absolute t-values) of text-based estimates and image-based estimates of similarity in predicting linguistic ratings of similarity (left) and visual ratings of similarity (right) for word pairs, as obtained from De Groot et al. (2016). Asterisks indicate significant associations between the model-based estimates and similarity ratings. 
176) $=.753 ; \mathrm{p}=.387 ; \mathrm{r}$-squared change $=.004)$ Results from this model showed that only image-based estimates significantly predict ratings of vision-based similarity $(\mathrm{t}(176)=3.261 ; \mathrm{p}=.001)$, while the effect of text-based similarity was not found to be significant $(\mathrm{t}(176)=-.868 ; \mathrm{p}=.387)$ (Figure 4). This result clearly demonstrates that (only) image-based estimates capture human intuitions of vision-based similarity. Therefore, our results provide evidence for a double dissociation between image-based and textbased estimates in predicting human judgments of vision-based vis-à-vis language-based similarity.

Taken together, these findings demonstrate that image-based and text-based models capture the independent contribution that visual and linguistic properties of word-denoted items make to brain activation and human judgments about relatedness. Therefore, these findings indicate that image-based and text-based estimates are valid approximations of vision-based and language-based similarity between objects, respectively.

\section{Experimental section}

\section{Methods}

Behavioural database. A total of 1,111 primetarget pairs were extracted from the SPP mega-study (Hutchison et al., 2013) (RTs ms: M NT-short = 546.8; SD NT-short $=43.0 ;$ M NT-long $=533.7 ;$ SD NT-long = 45.9; M LDT-short = 662.5; SD LDT-short = 67.2; M LDT-long = 688.6; SD LDT-long = 69.4). The advantages of the mega-study approach, such as protecting from issues associated with low statistical power, are discussed and acknowledged in the literature (Keuleers \& Balota, 2015).

The priming paradigm requires participants to respond to a target item preceded by a prime item. In the present study, we employed 4 different variants of the priming paradigm: NT short-SOA; LDT shortSOA; NT long-SOA; LDT long-SOA. Due to the nature of the task, the LDT included both target words and target non-words, and the task consisted of responding whether a presented target was a word or not. The NT included only words, and it required participants to read aloud the presented targets. For each of these 4 conditions, reaction time data for 1,661 target words, each preceded by 4 different primes, were retrieved. Data were collected from 768 English speakers in total, and each item received responses from approximately 32 subjects. We analyzed the pairaggregated data provided by Hutchison et al (2013), resulting in a single data point for each primetarget pair for each of the 4 task conditions. Pairs were selected from the full database following four criteria: first, both target and prime needed to be included in the text-based model available on SNAUT (Mandera et al., 2017); second, both target and prime needed to be associated to at least 100 pictures in ImageNet (Deng et al., 2009), a database of over 14 million labelled high-resolution images organized in about 20,000 categories (updated on April 30, 2010 http://image-net.org/about-stats); third, both target and prime needed to be associated to distinct categories in ImageNet (e.g., the pair beast-animal was excluded because both words were tags for the same category, and as a result associated with the same set of images). Finally, from this dataset, we extracted data for wordpairs for which all lexical measures in our analysis were available (see the following section).

However, the SPP item set (Hutchison et al., 2013) was not explicitly devised to capture perceptual aspects of the word-denoted objects. To ensure that our selected items covered a visual similarity range comparable with the standard in the perceptualpriming literature, we hence compared the variability in vision-based similarity estimates for our item pairs with the one for item pairs obtained from a dataset specifically designed to capture a wide distribution of visual similarity (Pecher et al., 1998). Following the method of Pecher et al. (1998), we generated 1000 different variants of such an item set. Each variant included the same 29 related item-pairs (e.g., orangeball, pistol-drill) along with a varying set of 29 unrelated item pairs (e.g., orange-coffee, pistol-ball), obtained by randomly pairing the words comprised in the related pairs (see section "Validation of the computational systems" for more details). We found that the variability in vision-based similarity in Pecher et al.'s items (mean interquartile range $=0.164 \pm$ $0.015 ; \min =0.387 ; \max =0.964)$ is not higher than the variability in vision-based similarity between the SPP item pairs employed in the present study (interquartile range $=0.213 ; \min =0.351 ; \max =$ 0.998) - if anything, it is lower. Therefore, the results of these simulations demonstrate that our item set captures a range of vision-based similarity that is 
comparable to the standard in the perceptual-priming literature.

Analyses. For each prime-target pair, we computed estimates of vision-based and language-based similarity. Note that although vision-based and language-based similarity were here estimated from independent sources of data (i.e., through computational models trained on text corpora and image databases), their correlation was high and significant $(\mathrm{r}(1109)=.50, \mathrm{p}<.001)$. Then, we tested for each experimental condition whether vision-based similarity explains variance in behavioural responses to linguistic stimuli over and above language-based similarity. To this end, we conducted three-step hierarchical regression analyses on response times for the four experimental conditions. In the first step (baseline model) we fitted a model with logarithmically transformed reaction times as the dependent variable and, as predictors, prime orthographic length, target orthographic length, logarithmically transformed prime and target frequencies (SUBTLEX-US corpus; Brysbaert \& New, 2009), logarithmically transformed prime and target contextual diversity (SUBTLEX-US corpus; Brysbaert \& New, 2009), a measure of orthographic neighbourhood (OLD20; Yarkoni, Balota, \& Yap, 2008), a measure of phonological neighbourhood (PLD20; Suárez, Tan, Yap, \& Goh, 2011), and the number of syllables. These lexical variables were included in the baseline model to account for the impact of word-specific properties, which are independent of the prime-target relation but associated with the mere processing of the word stimulus. After having established a baseline for each condition, we added the language-based similarity estimates (linguistic model) and assessed whether this additional parameter improved the model fit. Finally, we added to this model the vision-based similarity estimates (visual model) to assess whether vision-based similarity explains variance in behavioural responses to linguistic stimuli over and above language-based similarity (all item materials, data, and analysis scripts are available at https://osf.io/n7gqa/).

Note that by including language-based similarity first, we gave strict priority to linguistic effects before considering vision-based effects. As a consequence, in the comparison between the second to the third step of the hierarchical model comparison procedure, any shared variance between the variables is already captured by the language-based similarity estimates. This procedure may potentially underestimate visionbased similarity effects. However, we took this conservative approach in evaluating vision-based similarity effects because language-based similarity effects are well-established in the priming literature (Günther et al., 2016; Jones et al., 2006; Mandera et al., 2017) and previous research has concluded that visual effects do not exist (Pecher et al., 1998; Hutchison, 2003). Therefore, it needs to be demonstrated that purely visual information has any predictive power that is not already captured by linguistic information.

\section{Results}

Effects of language-based and vision-based estimates. The comparisons between the baseline model and the linguistic model indicated that language-based similarity improved the baseline model fit in all conditions (LDTshort-SOA: $\mathrm{p}<.001$; LDTlong-SOA: $\mathrm{p}<.001$; NTlong-SOA: $\mathrm{p}<.001)$, except for NTshort-SOA $(\mathrm{p}=.158)$. This effect of language-based similarity on reaction times was facilitatory (LDTshort-SOA: $\mathrm{p}<.001$; LDTlongSOA: $\mathrm{p}<.001$; NTlong-SOA: $\mathrm{p}<.001)$. The inclusion of vision-based similarity in the regression model resulted in a significant improvement in goodness-offit in the LDTshort-SOA condition $(\mathrm{p}=.002)$. Visionbased similarity had a facilitatory effect on reaction times $(\mathrm{p}=.002)$. No improvement in the model fit was observed in the other conditions: LDTlong-SOA ( $\mathrm{p}=$ .133); NTlong-SOA ( $\mathrm{p}=.671)$; NTshort-SOA ( $\mathrm{p}=$ .569) (Figure 5). The pattern of the language-based effects was consistent between the linguistic and the visual regression models. The interaction between vision-based similarity and condition, when considering the pooled dataset, was significant $(\mathrm{F}(3$, $3327)=7.421 ; \mathrm{p}<.001)$, indicating that the perceptual effect is indeed different across the four conditions. Results of the baseline, linguistic, and visual regression models per condition, including parameters for both language and vision-based similarity, are displayed in Table 1. 


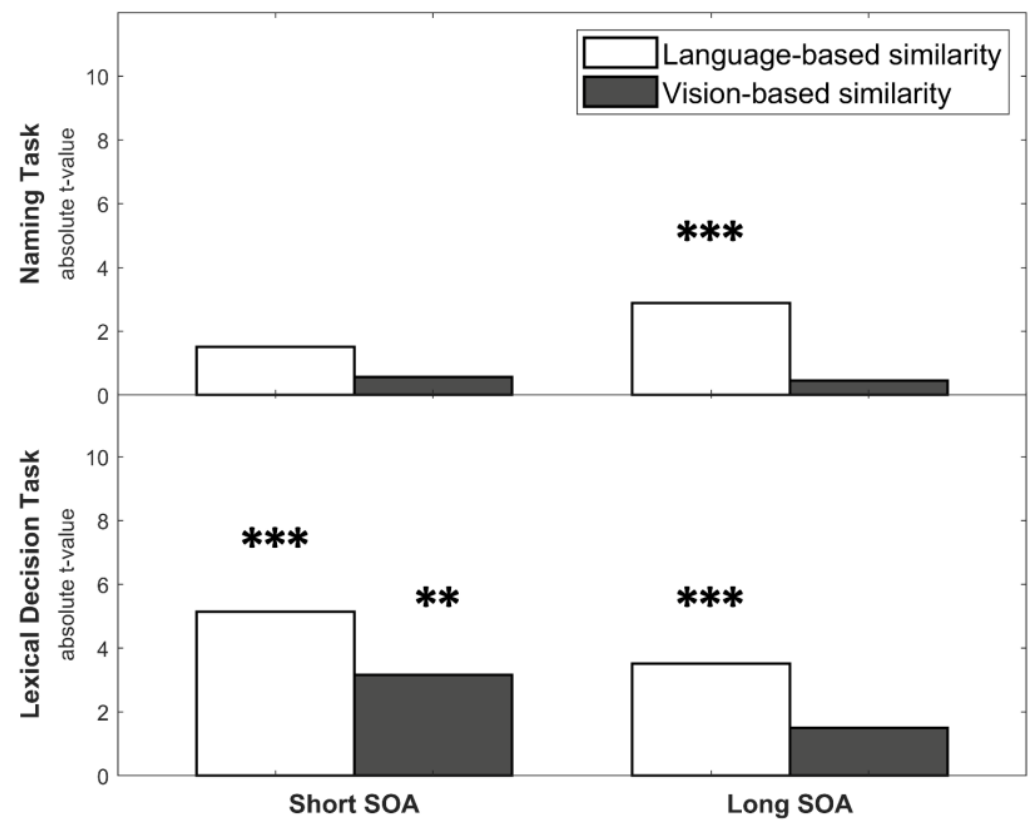

Note: $* \mathrm{p}<.05 ; * * \mathrm{p}<.01 ; * * * \mathrm{p}<.001$

Fig 5: Strength of the effects of language-based and vision-based similarity on the logarithmic RTs in absolute t-values for each of the SOAs (short-SOA $=200 \mathrm{~ms}$; long-SOA $=1200 \mathrm{~ms}$ ) and tasks (Lexical Decision Task and Naming Task). Asterisks indicate significant associations between the considered similarity and log-transformed RTs. 


\begin{tabular}{|c|c|c|c|c|}
\hline & \multicolumn{2}{|c|}{ Naming Task } & \multicolumn{2}{|c|}{ Lexical Decision Task } \\
\hline & $\begin{array}{l}\text { Short } \\
\text { SOA }\end{array}$ & $\begin{array}{l}\text { Long } \\
\text { SOA }\end{array}$ & $\begin{array}{l}\text { Short } \\
\text { SOA }\end{array}$ & $\begin{array}{l}\text { Long } \\
\text { SOA }\end{array}$ \\
\hline \multicolumn{5}{|c|}{ Step 1 (baseline model) } \\
\hline Adjusted $\mathrm{R}^{2}$ & .086 & .060 & 209 & .157 \\
\hline $\mathrm{F}(12,1098)$ & $9.720 * * *$ & $6.910 * * *$ & $25.380 * * *$ & $18.280^{* * *}$ \\
\hline \multicolumn{5}{|c|}{ Step 2 (linguistic model) } \\
\hline Language-based similarity & -1.414 & $-3.589 * * *$ & $-7.755 * * *$ & $-4.934 * * *$ \\
\hline Adjusted $\mathrm{R}^{2}$ & .087 & .070 & .249 & .175 \\
\hline $\mathrm{F}(13,1097)$ & $9.134 * * *$ & 7.438 *** & $29.310^{* * *}$ & $19.110 * * *$ \\
\hline \multicolumn{5}{|c|}{ Model Comparison (language vs. baseline): } \\
\hline Adjusted $\Delta \mathrm{R}^{2}$ & .0008 & .0101 & .0405 & .0175 \\
\hline $\mathrm{F}(1,1097)$ & 2.000 & $12.879 * * *$ & 60.148*** & 24.339*** \\
\hline \multicolumn{5}{|c|}{$\underline{\text { Step } 3 \text { (visual model) }}$} \\
\hline Vision-based similarity & .570 & -.425 & $-3.160 * *$ & -1.505 \\
\hline Language-based similarity & -1.509 & $-2.887 * *$ & $-5.141 * * *$ & $-3.509 * * *$ \\
\hline Adjusted $\mathrm{R}^{2}$ & .086 & .069 & .255 & .176 \\
\hline $\mathrm{F}(14,1096)$ & $8.499 * * *$ & $6.915^{* * *}$ & $28.150 * * *$ & $17.920 * * *$ \\
\hline \multicolumn{5}{|c|}{ Model Comparison (vision vs. language): } \\
\hline Adjusted $\Delta \mathrm{R}^{2}$ & -.0006 & -.0007 & .0061 & .0010 \\
\hline $\mathrm{F}(1,1096)$ & .325 & .181 & 9.988** & 2.266 \\
\hline
\end{tabular}

Table 1: Results (F Test, $\mathrm{R}^{2}$ values, and $\mathrm{t}$-values for vision-based and language-based similarity estimates) for each hierarchical step of the regression model predicting log-transformed RTs for each of the SOA (short-SOA $=200 \mathrm{~ms}$; long-SOA $=1200 \mathrm{~ms}$ ) and task (Lexical Decision Task and Naming Task) conditions. Step 1 (baseline model) predictors: lexical variables. Step 2 (linguistic model) predictors: lexical variables + language-based similarity. Step 3 (visual model) predictors: lexical variables + language-based similarity + vision-based similarity. Asterisks indicate significant association with logarithmic RT.

Vision-based model robustness. After evaluating the perceptual impact on priming data, we assessed the robustness of the effects by testing whether the obtained results were associated to a single, specific modelling set-up or rather held across a range of different architectures and representations (compare Zhang et al., 2018).

First, we investigated the robustness of our results when based on different representations from the same architecture, that is, when visual activations were computed based on different layers of the VGG-F model (Chatfield et al., 2014). Previous research suggests that the various layers of the model encode different types of visual features (from low-level to high-level visual features as the hierarchical level of the model increases; LeCun, Bengio, \& Hinton, 2015). Thus, we examined whether the observed effects held when other layers of the model were considered. We tested, for each layer ranging from the first to the sixth, whether vision-based similarity estimates (i.e., cosine similarities between the distributional activations of primes and targets) predicted RTs in semantic priming over and above language-based similarity (see Table 2). Consistently with the results for layer 7 , none of these vision-based similarity estimates improved the model fit in the NT with short-SOA (all ps > .311), in the NT with long-SOA (all ps > .317), and in the LDT with long-SOA (all ps > .133). On the other hand, vision-based similarity estimates across all layers improved the model fit in the LDT with short-SOA (all ps < .028). These results suggest that vision-based effects can be observed both in terms of similarity in abstract visual representations of word pairs (learned at deeper layers of the network) as well as in terms of similarity in basic visual features of word-denoted items, which are represented at shallower layers of the network (LeCun et al., 2015; Smith et al., 2017; Zeiler $\&$ Fergus, 2014). 


\begin{tabular}{|c|c|c|c|c|}
\hline & \multicolumn{2}{|c|}{ Naming Task } & \multicolumn{2}{|c|}{ Lexical Decision Task } \\
\hline & $\begin{array}{l}\text { Short } \\
\text { SOA }\end{array}$ & $\begin{array}{l}\text { Long } \\
\text { SOA }\end{array}$ & $\begin{array}{l}\text { Short } \\
\text { SOA }\end{array}$ & $\begin{array}{l}\text { Long } \\
\text { SOA }\end{array}$ \\
\hline \multicolumn{5}{|c|}{$\frac{\text { Step } 3 \text { (visual model) }}{\text { Laver: } 1}$} \\
\hline Vision-based similarity & -.445 & 1.000 & $-2.460 *$ & -.133 \\
\hline Language-based similarity & -1.157 & $-\mathbf{3 . 7 0 8} * * *$ & $-6.359 * * *$ & $-4.550 * * *$ \\
\hline Adjusted $\mathrm{R}^{2}$ & .086 & .070 & .253 & .174 \\
\hline $\mathrm{F}(14,1096)$ & $8.489 * * *$ & $6.979 * * *$ & $27.780 * * *$ & $17.730 * * *$ \\
\hline \multicolumn{5}{|c|}{ Model Comparison (vision vs. language): } \\
\hline Adjusted $\Delta \mathrm{R}^{2}$ & -.0007 & $<.0001$ & .0034 & -.0007 \\
\hline $\mathrm{F}(1,1096)$ & .198 & 1.001 & $6.050 *$ & .018 \\
\hline \multicolumn{5}{|c|}{ Layer: 2} \\
\hline Vision-based similarity & .520 & -.538 & $-2.829 * *$ & .307 \\
\hline Language-based similarity & -1.506 & $-3.206^{* *}$ & $-6.402 * * *$ & $-4.754 * * *$ \\
\hline Adjusted $\mathrm{R}^{2}$ & .086 & .070 & .254 & .174 \\
\hline $\mathrm{F}(14,1096)$ & $8.495 * * *$ & $6.923 * * *$ & $27.960 * * *$ & $17.730 * * *$ \\
\hline \multicolumn{5}{|c|}{ Model Comparison (vision vs. language): } \\
\hline Adjusted $\Delta \mathrm{R}^{2}$ & -.0006 & -.0006 & .0047 & -.0007 \\
\hline $\mathrm{F}(1,1096)$ & .271 & .290 & $8.001 * *$ & .094 \\
\hline \multicolumn{5}{|c|}{ Layer: 3} \\
\hline Vision-based similarity & .739 & .029 & $-2.842 * *$ & -.909 \\
\hline Language-based similarity & -1.592 & $-3.296 * *$ & $-5.982 * * *$ & $-4.151 * * *$ \\
\hline Adjusted $\mathrm{R}^{2}$ & .087 & .069 & .254 & .175 \\
\hline $\mathrm{F}(14,1096)$ & $8.517 * * *$ & $6.901 * * *$ & $27.970 * * *$ & $17.800 * * *$ \\
\hline \multicolumn{5}{|c|}{ Model Comparison (vision vs. language): } \\
\hline Adjusted $\Delta \mathrm{R}^{2}$ & -.0004 & -.0008 & .0048 & -.0001 \\
\hline $\mathrm{F}(1,1096)$ & .546 & .001 & $8.077 * *$ & .827 \\
\hline \multicolumn{5}{|c|}{ Layer: 4} \\
\hline Vision-based similarity & 1.014 & -.308 & $-2.241^{*}$ & -1.199 \\
\hline Language-based similarity & -1.715 & $-3.090 * *$ & $-6.002 * * *$ & $-3.910 * * *$ \\
\hline Adjusted $\mathrm{R}^{2}$ & .087 & .069 & .252 & .175 \\
\hline $\mathrm{F}(14,1096)$ & $8.555 * * *$ & $6.908 * * *$ & $27.680 * * *$ & $17.850 * * *$ \\
\hline \multicolumn{5}{|c|}{ Model Comparison (vision vs. language): } \\
\hline Adjusted $\Delta \mathrm{R}^{2}$ & $<.0001$ & -.0008 & .0027 & .0003 \\
\hline $\mathrm{F}(1,1096)$ & 1.028 & .095 & $5.023 *$ & 1.439 \\
\hline \multicolumn{5}{|c|}{ Layer: 5} \\
\hline Vision-based similarity & .714 & -.720 & $-2.207^{*}$ & -1.171 \\
\hline Language-based similarity & -1.584 & $-2.879 * *$ & $-5.943 * * *$ & $\mathbf{- 3 . 8 7 8} * * *$ \\
\hline Adjusted $\mathrm{R}^{2}$ & .087 & .070 & .252 & .175 \\
\hline $\mathrm{F}(14,1096)$ & $8.514 * * *$ & $6.941 * * *$ & $27.660 * * *$ & $17.840 * * *$ \\
\hline \multicolumn{5}{|c|}{ Model Comparison (vision vs language): } \\
\hline Adjusted $\Delta \mathrm{R}^{2}$ & -.0004 & -.0004 & .0026 & .0003 \\
\hline $\mathrm{F}(1,1096)$ & .510 & .519 & $4.871 *$ & 1.372 \\
\hline \multicolumn{5}{|c|}{ Layer: 6} \\
\hline Vision-based similarity & .857 & -.744 & $-2.254 *$ & -.877 \\
\hline Language-based similarity & -1.652 & $-2.768 * *$ & $-5.680 * * *$ & $-3.877 * * *$ \\
\hline Adjusted $\mathrm{R}^{2}$ & .087 & .070 & .252 & .175 \\
\hline $\mathrm{F}(14,1096)$ & $8.532 * * *$ & $6.944 * * *$ & $27.680 * * *$ & $17.790 * * *$ \\
\hline \multicolumn{5}{|c|}{ Model Comparison (vision vs language): } \\
\hline Adjusted $\Delta \mathrm{R}^{2}$ & -.0002 & -.0004 & .0028 & .0002 \\
\hline$F(1,1096)$ & .734 & .554 & $5.081^{*}$ & .770 \\
\hline
\end{tabular}

Table 2: Results (F Test, R2 values, and t-values of vision-based similarity) for step 3 of the regression model are reported for layers 1-6. Asterisks indicate significant associations with logarithmic RT. 
Second, we tested to what extent the present pattern of results was robust when changing the computational architecture used to compute visual similarity. That is, we ran a series of analyses to exclude the possibility that the obtained effect depended on the specific visual model adopted in the present study. Literature reports various prominent deep convolutional networks for visual recognition. Here, in addition to the VGG-F model (Chatfield et al., 2014), we tested the robustness of our predictions with three other different CNNs: VGG-16, VGG-19 (Simonyan \& Zisserman, 2014), and AlexNet (Krizhevsky et al., 2012). As reported in Table 3, results are highly consistent across all the different architectures we tested (i.e., vision-based similarity estimated for each model predicted RTs only in LDT with short-SOA and not in other conditions), suggesting that the perceptual facilitation effect we found cannot be ascribed to the specificity of a single model.

All item materials, data, and analysis scripts are available at https://osf.io/n7gqa/.

\begin{tabular}{|c|c|c|c|c|}
\hline & \multicolumn{2}{|c|}{ Naming Task } & \multicolumn{2}{|c|}{ Lexical Decision Task } \\
\hline & $\begin{array}{l}\text { Short } \\
\text { SOA }\end{array}$ & $\begin{array}{l}\text { Long } \\
\text { SOA }\end{array}$ & $\begin{array}{l}\text { Short } \\
\text { SOA }\end{array}$ & $\begin{array}{l}\text { Long } \\
\text { SOA }\end{array}$ \\
\hline \multicolumn{5}{|c|}{ Step 3 (visual model) } \\
\hline Vision-based similarity & .551 & -.558 & $-2.894 * *$ & -1.612 \\
\hline Language-based similarity & -1.493 & $-2.765^{* *}$ & $-5.115 * * *$ & $-\mathbf{3 . 3 6 3} * * *$ \\
\hline Adjusted $\mathrm{R}^{2}$ & .086 & .070 & .254 & .176 \\
\hline$F(14,1096)$ & $8.498 * * *$ & $6.925 * * *$ & $28.000 * * *$ & $17.950 * * *$ \\
\hline \multicolumn{5}{|c|}{ Model Comparison (vision vs. language): } \\
\hline Adjusted $\Delta \mathrm{R}^{2}$ & -.0006 & -.0006 & .0050 & .0012 \\
\hline $\mathrm{F}(1,1096)$ & .304 & .311 & $8.376^{* *}$ & 2.597 \\
\hline \multicolumn{5}{|c|}{ CNN: VGG-19 } \\
\hline Vision-based similarity & .535 & -.507 & $-2.836 * *$ & -1.675 \\
\hline Language-based similarity & -1.484 & $-2.784^{* *}$ & $-5.123 * * *$ & $-\mathbf{3 . 3 1 7} * * *$ \\
\hline Adjusted $\mathrm{R}^{2}$ & .086 & .069 & .254 & .176 \\
\hline$F(14,1096)$ & $8.496^{* * * *}$ & $6.921 * * *$ & $27.970 * * *$ & $17.970 * * *$ \\
\hline \multicolumn{5}{|c|}{ Model Comparison (vision vs. language): } \\
\hline Adjusted $\Delta \mathrm{R}^{2}$ & -.0006 & -.0006 & .0048 & .0014 \\
\hline $\mathrm{F}(1,1096)$ & .286 & .257 & $8.041^{* *}$ & 2.805 \\
\hline \multicolumn{5}{|c|}{ CNN: AlexNet } \\
\hline Vision-based similarity & $.583-$ & -.348 & $-3.277 * *$ & -1.656 \\
\hline Language-based similarity & -1.515 & $-2.924 * *$ & $-\mathbf{5 . 0 8 0} * * *$ & $\mathbf{- 3 . 4 3 2} * * *$ \\
\hline Adjusted $\mathrm{R}^{2}$ & .086 & .069 & .256 & .176 \\
\hline$F(14,1096)$ & $8.501 * * *$ & $6.910 * * *$ & $28.230 * * *$ & $17.960 * * *$ \\
\hline \multicolumn{5}{|c|}{ Model Comparison (vision vs. language): } \\
\hline Adjusted $\Delta \mathrm{R}^{2}$ & -.0005 & -.0007 & .0066 & 0013 \\
\hline $\mathrm{F}(1,1096)$ & .340 & .121 & $10.738 * *$ & 2.742 \\
\hline
\end{tabular}

Note. $* \mathrm{p}<.05 ; * * \mathrm{p}<.01 ; * * * \mathrm{p}<.001$

Table 3: Results (F Test, $\mathrm{R}^{2}$ values, and $\mathrm{t}$-values of vision-based similarity) for the step 3 of the regression model are reported for 3 different CNN architectures:VGG-16, VGG-19 (Simonyan \& Zisserman, 2014) and AlexNet (Krizhevsky et al., 2012). Asterisks indicate significant association with logarithmic RT. 


\section{Discussion}

In the present study, we exploited data-driven computational models to reveal the potential contribution of perceptual information (here in the form of visual simulation) in the conceptual processing stream. To this aim, we tested in a largescale behavioural dataset whether semantic priming effects for word pairs are influenced by the visual properties of the objects they denote, as approximated by a data-driven convolutional neural network. Using this approach, we found that vision-based similarity estimates can predict human performance over and above their linguistic counterparts in a wordprocessing task where visual processing is neither solicited nor required. This finding suggests that even in such a purely linguistic task, the activation of perceptual information (or perceptual simulation) of the prime automatically influences processing and semantic access to the target word (in line with Barsalou, 2008). Importantly, this finding reconciles behavioural results on semantic priming with several studies in the neuroimaging and neuropsychological literature, which attribute to perceptual simulation a role in facilitating conceptual processing (Hauk et al., 2004; Trumpp et al., 2013; for review and discussion see, Binder \& Desai, 2011 and Pulvermüller, 2018).

It is worth noting that we were particularly conservative in evaluating visual effects by first controlling for language-based similarity. This means that we took into consideration a somewhat restricted portion of visual experience and, specifically, the portion of visual experience that cannot be reduced to language data. In fact, it is known that, since we can talk about objects that we perceive, language data end up containing visual information (Andrews et al., 2009; Connolly et al., 2007; Johns \& Jones, 2012; Louwerse, 2007, 2011; see also the positive correlation that we found between vision-based and language-based similarity in the Analyses section). Here we opted for such an approach since the aim of the present work is to provide evidence for an effect (perceptual priming) that is largely challenged in the literature. In this scenario, we believe that being as conservative as possible is of the utmost importance. Indeed, our approach puts potential effects of our vision-based estimates under strict scrutiny, ensuring that any possible effect of vision-based similarity emerged here could not be accounted for by lexicalorthographic aspects. Importantly, the present results indicate that, even under such a conservative procedure, visual experience can predict semantic priming effects.

However, our results also indicate that vision-based similarity does not consistently affect word processing across all the conditions analyzed here. Indeed, visionbased similarity only predicts early semantic priming effects (at the 200-ms SOA) in the LDT, which is known to be, overall, more sensitive to substantial semantic-level effects than the NT (Lucas, 2000; but see Connell \& Lynott, 2014, for different results), while we observed language-based similarity effects also at later stages (1200 ms SOA) in both the NT and the LDT. These latter results are not compatible with the claim of a complete equivalence between word comprehension and sensorimotor simulation (e.g., Glenberg, 2015): in that case, we would have expected no experimental condition where we observe linguistic effects without visual influence on semantic priming. Instead, our results indicate a dissociation between language- and vision-based semantic effects.

Taken together, our results support the grounded view suggesting an impact of perceptual experience in language processing (Barsalou, 2008). This emerges even if such a piece of information is not necessarily required by the task at hand-in a lexical decision task, participants have to indicate whether or not a word exists, an aspect which could in principle be judged at the word-form level alone. However, previous research has demonstrated that semantic factors routinely affect lexical decisions (Balota et al., 2007; Lucas, 2000; Seidenberg \& McClelland, 1989). In the present study, we demonstrate that this unsolicited meaning-level activation entails the activation of perceptual information, thus shedding new light on previous claims that such information is only accessed when required by the task (Lebois et al., 2015, Ostarek \& Huettig, 2017, 2019). Employing an adequate computational framework to investigate a large-scale dataset allows us to uncover these automatic activations of perceptual information. This finding is also relevant in light of the debate on the functional relevance of sensorimotor activation (see Barsalou et al., 2003; Mahon \& Caramazza, 2008; Strozyk et al., 2019): while our results remain agnostic to whether sensorimotor activation is indeed necessary for 
processing, the observed facilitatory effects of visionbased similarity in a linguistic task shows that this activation is automatic and useful for processing.

At the same time, our results scale down the weight attributed to perceptual simulation by strong grounded accounts (as discussed in Mahon, 2015b; see Glenberg, 2015). While we observe that the visual features of the object connoted by a previously presented word can ease the processing of a following lexical item in certain experimental settings, our results also suggest that perceptual simulation is not equivalent to word comprehension. Otherwise, the facilitatory impact of vision-based similarity would have paralleled or even eclipsed the language-based similarity effect across all the conditions considered. This is in line with the evidence that conceptual deficits in patients with sensory-motor impairments, when present, are mild and not "catastrophic", as would be predicted by the strong grounded cognition hypothesis (Binder \& Desai, 2011; Mahon, 2015a; Mahon \& Caramazza, 2005).

Modelling similarity in a data-driven approach

Our study applies the potential of innovative computational approaches to the field of grounded cognition. The most crucial benefit of this method is that it allows us to characterize on a continuous scale the degree of similarity between prime and target pairs as derived from independent sources of data. Consequently, we were are able to consider a wide range of different item pairs contained in the semantic priming mega-study by Hutchison et al. (2013) rather than restricting our analyses to only a few extremes/dichotomized cases (i.e., "related" vs. "unrelated"; see also Baayen, 2004, for arguments against this practice). Over and above the evident benefit in terms of statistical power (Keuleers \& Balota, 2015), this approach also ensures that the obtained results reflect a larger sample of the lexicon of a speaker and are hence more generalizable. Moreover, this method protects against the problematic aspects that the adoption of human-based ratings of similarity as predictors has on the objectiveness and reliability of the study, and also avoids the epistemological loophole of predicting behavioural data (priming effects) from other behavioural data (ratings) (see Jones et al., 2015; Westbury, 2016). In the present study, the similarity between items is not based on human intuitions, but rather on collections of data that can be considered a proxy of the linguistic (text corpora) and perceptual (image databases) human experience. This experience serves as input to a learning model, whereas rating data are the outcome of a human learning process as filtered by ecologically questionable experimental instructions. This illustrates another important difference between the two cases: similarity ratings are artificially elicited behaviour that hardly exist outside of scientific studies, while corpora, on the other hand, consist of unelicited behaviour that occurs naturally in the outside world, and to which speakers are very frequently exposed (see Keuleers \& Marelli, 2020).

\section{Linguistic and sensorimotor activation in language processing}

Given our results, one may wonder why previous behavioural studies have not observed automatic effects of perceptual similarity. Over and above the much larger item set employed here, another difference is that most of these studies (Pecher et al., 1998; Schreuder et al., 1984) have employed an SOA between 350 and $400 \mathrm{~ms}$, which might be too long to detect visual effects under the assumption that visual activation is a fast-decaying process in word processing. Indeed, in the present study, we only observe a vision-based similarity effect at relatively short SOAs (200 ms). This fits with suggestions from previous works (Pecher et al., 1998; Schreuder et al., 1984), indicating that visual information is activated early in linguistic processing, and with neuroimaging studies showing that activation of sensorimotor systems occurs 200-300 ms after stimulus presentation (Hauk et al., 2004).

These findings have important implications for theories of embodied language processing, such as the language and simulated situation (LASS) theory (Barsalou et al., 2008) which specifies the role and time course of linguistic and sensorimotor activation during language processing. Explicitly going beyond a black-and-white view of whether cognition is grounded or not, this theory considers the relative contribution of different types of information. According to the LASS theory, lexical decision qualifies as a relatively shallow processing task, where access to meaning is limited, and sensorimotor activation is not necessary. Still, the theory assumes 
that processing of a linguistic stimulus such as a word always involves both linguistic and sensorimotor activation. Linguistic processing should take the lion's share, and the activation of linguistic information should peak first; however, the perceptual simulation system will also be activated, even if to a lesser degree and with a later peak. Concerning the other free parameters, the theory is under-specified: as stated by Barsalou et al., (2008), parameters such as the relative strength, peak, duration, and offset of activation are not assumed to be fixed yet are expected to change in response to different words and different task contexts (Barsalou et al., 2008, pp. 248-249).

Our findings support critical assumptions of the LASS theory: we observe perceptual activation in a lexical decision task, which is in line with the claim that sensorimotor activation is automatic. Further, this activation is observed in a short-SOA condition, in line with the claim that the activation is fast and early (see also Schreuder et al., 1984).

Importantly, however, our results in the lexical decision task also warrant a significant amendment to the LASS theory: while we observe linguistic effects at both short and long SOAs, in lexical decision, a visual effect is observed only at short but not at long SOAs. This implies that, in lexical decision, perceptual activation decays (a) earlier than linguistic activation and (b) before it can influence the processing of the target word (for which the earliest possible point is the onset of the target and the latest possible point is the execution of the response) in the long-SOA condition (at which point linguistic activation persists) 2 . Our results thus allow us to set clear boundaries for the offset of sensorimotor activation, which previously has been a free parameter of the LASS theory. Following the claim by Barsalou et al. (2008) that "when the cue is a word, contributions from the linguistic system precede those from the simulation system" (p. 248), the earlier offset of perceptual activation also directly implies that its duration is shorter than the duration of linguistic activation. As perceptual activation is not required and therefore does not need to be maintained for the actual

\footnotetext{
2 Note, however, that these implications cannot be generalized to tasks other than the lexical decision. Indeed, in property generation tasks, Barsalou's LASS theory would predict that sensorimotor simulations occur at later stages than linguistic processing (Barsalou
}

response, it is entirely in line with the LASS theory that perceptual activation lasts shorter and decays earlier.

However, at the same time, we also need to acknowledge that our results do not provide direct information concerning the relative onset of activation: since we observe both linguistic and perceptual activation at the shortest SOA, we can only conclude that both set in before the processing of the target word can be influenced in this condition. We can thus neither confirm nor reject the assumptions regarding the simultaneous onset of activation by Barsalou et al. (2003). Similarly, our study does not provide information about the peak or, in general, the time course of the strength of activation. However, it should be noted that our computational framework provides the toolkit to subject these open questions to empirical investigation. One possible approach to this issue is a systematic investigation of visual and linguistic priming effects at different SOAs (between $0 \mathrm{~ms}$ and 1,200 ms), which would allow for a detailed time-course analysis.

The impact of task requirements may also help in understanding the lack of visual effects in the NT. In fact, several authors suggested that access to the stimulus meaning has high relevance in the LDT because meaning itself is a useful dimension to consider in order to perform the task (i.e., to discriminate words from non-words) (e.g., Balota et al., 2004). Moreover, whereas the LDT requires access to, at least, the target lexical representation in order to be performed, the NT can, in principle, be carried out by solely relying on associations between peripheral linguistic units (graphemes and phonemes). Thus, in terms of the LASS theory, the NT would represent a shallower task than the LDT. As a result, in the NT, activation of the visual representation of the primedenoted object may be too limited to influence target processing. Accordingly, the literature indicates that semantic effects (including visual-semantic effects) tend to be overall smaller in the NT compared to the LDT (e.g., Balota et al., 2004; Chumbley \& Balota, 1984; other theoretical explanations for this effect in

et al., 2008), possibly because the sensorimotor representation in all its details is actively maintained to deal with task requirements. 
priming can be found in Lucas, 2000; see also Connell \& Lynott, 2014, for near equivalent/slightly larger semantic effects in NT vs. LDT).

A different explanation for the dissociation in vision-based effects found for the LDT vis-à-vis the NT may be related to differences in the attentional load of these tasks. As indicated by Lynott and Connell (2014), the NT (compared to the LDT) involves a higher attentional load by requiring both visual processing of word form and auditory processing of speech output. Following this consideration, in the NT, one would expect that attention involved in reading may interfere with the visual simulation of the word-denoted concept by leaving few resources free for simulation purposes (see Connell \& Lynott, 2012). As a result, when naming is combined with a semantic priming task, the visual simulation of the representation of the prime may be too limited to influence semantic processing of the target and, therefore, to allow, again, any substantial visual effect to emerge.

\section{Context and meaning}

Note that the semantic priming tasks employed here present items in isolation from any contextual information. In contrast, in everyday language use, word processing is always situated in specific contexts (e.g., in the context of other words or other sensorimotor information; Zwaan, 2014). Even if our study (alongside others, e.g., Lachmair et al., 2011; Thornton et al., 2012) suggests that such context is not necessarily required to elicit effects of sensorimotor activation, contextual information still has a considerable impact on mental representations. For example, the visual representation elicited when reading egg in the pan differs dramatically from egg in the fridge (Zwaan, Stanfield, \& Yaxley, 2002). At first glance, this seems to be incompatible with our approach, where each visual representation (and each linguistic representation) is modelled as a single fixed numerical vector. However, this characterization of the distributional approach is largely unjustified (see Günther et al., 2019, for a thorough discussion of this argument). For example, Kintsch (2001) has proposed a framework based on his construction-integration model (Kintsch, 1988) that updates the representation of a predicate based on its argument by additionally activating mutually similar representations. As a consequence, the meaning of shark will be different in This lawyer is a shark versus This fish is a shark (see Kintsch, 2000). Although this framework has been formulated for linguistic distributional vectors, the same principle can be applied to vision-based vectors.

In addition to on-line processing, context also plays a vital role in the acquisition of concepts and word meanings (Zwaan, 2014; Zwaan \& Madden, 2005). This aligns perfectly with our modelling approach, where contextual information has a decisive impact on the training of both the language- and the vision-based models used here. In fact, it plays a central role in distributional semantic models: these are based on the distributional hypothesis that postulates a correspondence (or even equivalence) between a word and the contexts in which it is used (Harris, 1954; Lenci, 2008). Accordingly, these models approximate word meanings through a statistical analysis of their contexts. At the same time, the computer-vision models employed here approximate visual entities through an analysis of co-occurrences between visual features. The training material of the visual model does not consist of only entities in front of a blank background, and the target object described by the label is not pre-identified during model training. Accordingly, visual scenes containing contextually similar objects will share visual features even if the objects themselves do not (Bruni et al., 2014): Images of ships, sharks, icebergs, oceans, and scuba usually contain a lot of blue, watery background. Similarly, visual scenes of wine almost always contain bottles, wine glasses, grapes, or a combination of these. Since the model is trained on visual features related to both the entity and its context, such as other entities in the same visual scene or the background, this context will, therefore, influence the resulting representations. As a result, these models provide a promising ground combining data-driven computational approaches with semantics and grounded cognition by building on the notion of context and the central role that it plays in both perspectives. 


\section{References}

Anderson, A. J., Bruni, E., Lopopolo, A., Poesio, M., \& Baroni, M. (2015). Reading visually embodied meaning from the brain: Visually grounded computational models decode visual-object mental imagery induced by written text. NeuroImage, 120, 309-322. https://doi.org/10.1016/j.neuroimage.2015.06.093

Anderson, J. R., \& Milson, R. (1989). Human Memory: An Adaptive Perspective. Psychological Review, 96(4), 703-719. https://doi.org/10.1037/0033-295X.96.4.703

Andrews, M., Frank, S., \& Vigliocco, G. (2014). Reconciling embodied and distributional accounts of meaning in language. Topics in Cognitive Science, 6(3), 359-370. https://doi.org/10.1111/tops.12096

Andrews, M., Vigliocco, G., \& Vinson, D. (2009). Integrating experiential and distributional data to learn semantic representations. Psychological Review, 116(3), 463. https://doi.org/10.1037/a0016261

Baayen, R. H. (2004). Statistics in psycholinguistics: A critique of some current gold standards. Mental Lexicon Working Papers, $1(1), 1-47$.

Balota, D. A., Cortese, M. J., Sergent-Marshall, S. D., Spieler, D. H., \& Yap, M. J. (2004). Visual word recognition of singlesyllable words. Journal of Experimental Psychology: General, 133(2), 283. https://doi.org/10.1037/0096-3445.133.2.283

Balota, D. A., Yap, M. J., Cortese, M. J., Hutchison, K. A., Kessler, B., Loftis, B., Neely, J. H., Nelson, D. L., Simpson, G. B., \& Treiman, R. (2007). The english lexicon project. In Behavior Research Methods (Vol. 39, Issue 3, pp. 445-459). Springer New York LLC. https://doi.org/10.3758/BF03193014

Baroni, M., Dinu, G., \& Kruszewski, G. (2014). Don't count, predict! A systematic comparison of context-counting vs. context-predicting semantic vectors. In $\mathrm{K}$. Toutanova \& $\mathrm{H}$. Wu (Eds.), Proceedings of the 52nd Annual Meeting of the Association for Computational Linguistics (pp. 238-247). Association for Computational Linguistics. https://doi.org/10.3115/v1/P14-1023

Barsalou, L. W. (2008). Grounded Cognition. Annual Review of Psychology, 59(1), 617-645. https://doi.org/10.1146/annurev.psych.59.103006.093639

Barsalou, L. W., Santos, A., Simmons, W. K., \& Wilson, C. D. (2008). Language and simulation in conceptual processing. Symbols, Embodiment, and Meaning, 245-283. https://doi.org/10.1093/acprof:oso/9780199217274.003.0013

Barsalou, L. W., Simmons, W. K., Barbey, A. K., \& Wilson, C. D. (2003). Grounding conceptual knowledge in modality-specific systems. Trends in Cognitive Sciences, 7(2), 84-91 https://doi.org/10.1016/S1364-6613(02)00029-3
Bhatia, S. (2017). Associative judgment and vector space semantics Psychological Review, 124(1), 1-20. https://doi.org/10.1037/rev0000047

Binder, J. R., \& Desai, R. H. (2011). The neurobiology of semantic memory. Trends in Cognitive Sciences, 15(11), 527-536. https://doi.org/10.1016/j.tics.2011.10.001

Bracci, S., Ritchie, J. B., Kalfas, I., \& Op de Beeck, H. P. (2019). The Ventral Visual Pathway Represents Animal Appearance over Animacy, Unlike Human Behavior and Deep Neural Networks. The Journal of Neuroscience : The Official Journal of the Society for Neuroscience, 39(33), 6513-6525. https://doi.org/10.1523/JNEUROSCI.1714-18.2019

Bruni, E., Tran, N. K., \& Baroni, M. (2014). Multimodal Distributional Semantics. In Journal of Artificial Intelligence Research (Vol. 49). https://doi.org/10.1613/jair.4135

Brysbaert, M., \& New, B. (2009). Moving beyond Kučera and Francis: A critical evaluation of current word frequency norms and the introduction of a new and improved word frequency measure for American English. Behavior Research Methods, 41(4), 977-990. https://doi.org/10.3758/BRM.41.4.977

Bullinaria, J. A., \& Levy, J. P. (2007). Extracting semantic representations from word co-occurrence statistics: A computational study. Behavior Research Methods, 39(3), 510526. https://doi.org/10.3758/BF03193020

Chatfield, K., Simonyan, K., Vedaldi, A., \& Zisserman, A. (2014). Return of the devil in the details: Delving deep into convolutional nets. ArXiv Preprint ArXiv:1405.3531.

Chumbley, J. I., \& Balota, D. A. (1984). A word's meaning affects the decision in lexical decision. Memory \& Cognition, 12(6), 590-606. https://doi.org/10.3758/BF03213348

Connell, L., \& Lynott, D. (2012). When does perception facilitate or interfere with conceptual processing? The effect of attentional modulation. Frontiers in Psychology, 3(NOV). https://doi.org/10.3389/fpsyg.2012.00474

Connell, L., \& Lynott, D. (2014). I see/hear what you mean Semantic activation in visual word recognition depends on perceptual attention. Journal of Experimental Psychology: General, 143(2), 527. https://doi.org/10.1037/a0034626

Connolly, A. C., Gleitman, L. R., \& Thompson-Schill, S. L. (2007), Effect of congenital blindness on the semantic representation of some everyday concepts. Proceedings of the National Academy of Sciences, 104(20), 8241-8246. https://doi.org/10.1073/pnas.0702812104

De Groot, F., Koelewijn, T., Huettig, F., \& Olivers, C. N. L. (2016) A stimulus set of words and pictures matched for visual and semantic similarity. Journal of Cognitive Psychology, 28(1), 115. https://doi.org/10.1080/20445911.2015.1101119

Deng, J., Dong, W., Socher, R., Li, L.-J., Li, K., \& Fei-Fei, L. 
(2009). Imagenet: A large-scale hierarchical image database. Computer Vision and Pattern Recognition, 2009. CVPR 2009. IEEE Conference On, 248-255. https://doi.org/10.1109/CVPR.2009.5206848

Ferraresi, A., Zanchetta, E., Baroni, M., \& Bernardini, S. (2008). Introducing and evaluating $\mathrm{ukWaC}$, a very large web-derived corpus of English. Proceedings of the 4th Web as Corpus Workshop (WAC-4) Can We Beat Google, 47-54.

Fischer, M. H. (2012). A hierarchical view of grounded, embodied, and situated numerical cognition. Cognitive Processing, 13(1 SUPPL). https://doi.org/10.1007/s10339-012-0477-5

Flores D’Arcais, G. B., Schreuder, R., \& Glazenborg, G. (1985). Semantic activation during recognition of referential words. Psychological Research, 47(1), 39-49. https://doi.org/10.1007/BF00309217

Glenberg, A. M. (2015). Few believe the world is flat: How embodiment is changing the scientific understanding of cognition. Canadian Journal of Experimental Psychology/Revue Canadienne de Psychologie Expérimentale, 69(2), 165. https://doi.org/10.1037/cep0000056

Glenberg, A. M., \& Kaschak, M. P. (2002). Grounding language in action. Psychonomic Bulletin \& Review, 9(3), 558-565. https://doi.org/10.3758/bf03196313

Günther, F., Dudschig, C., \& Kaup, B. (2016). Latent semantic analysis cosines as a cognitive similarity measure: Evidence from priming studies. Quarterly Journal of Experimental Psychology, 69(4), 626-653. https://doi.org/10.1080/17470218.2015.1038280

Günther, F., Petilli, M. A., \& Marelli, M. (2020). Semantic transparency is not invisibility: A computational model of perceptually-grounded conceptual combination in word processing. Journal of Memory and Language, 112, 104104. https://doi.org/10.1016/J.JML.2020.104104

Günther, F., Petilli, M. A., Vergallito, A., \& Marelli, M. (2020). Images of the unseen: Extrapolating visual representations for abstract and concrete words in a data-driven computational model. Psychological Research. https://doi.org/10.1007/s00426-020-01429-7

Günther, F., Rinaldi, L., \& Marelli, M. (2019). Vector-Space Models of Semantic Representation From a Cognitive Perspective: A Discussion of Common Misconceptions. Perspectives on Psychological Science, 14(6), 1006-1033. https://doi.org/10.1177/1745691619861372

Han, J., Kamber, M., \& Pei, J. (2012). Data Mining: Concepts and Techniques. In Data Mining: Concepts and Techniques. Elsevier Inc. https://doi.org/10.1016/C2009-0-61819-5

Harris, Z. S. (1954). Distributional structure. Word, 10(2-3), 146162. https://doi.org/10.1080/00437956.1954.11659520
Hauk, O., Johnsrude, I., \& Pulvermüller, F. (2004). Somatotopic representation of action words in human motor and premotor cortex. Neuron, 41(2), 301-307. https://doi.org/10.1016/s08966273(03)00838-9

Hempel, C. G., \& Oppenheim, P. (1948). Studies in the Logic of Explanation. Philosophy of Science, 15(2), 135-175. https://doi.org/10.1086/286983

Hollis, G. (2017). Estimating the average need of semantic knowledge from distributional semantic models. Memory and Cognition, 45(8), 1350-1370. https://doi.org/10.3758/s13421017-0732-1

Hutchison, K. A. (2003). Is semantic priming due to association strength or feature overlap? A microanalytic review. Psychonomic Bulletin \& Review, 10(4), 785-813. https://doi.org/10.3758/bf03196544

Hutchison, K. A., Balota, D. A., Neely, J. H., Cortese, M. J., CohenShikora, E. R., Tse, C. S., Yap, M. J., Bengson, J. J., Niemeyer, D., \& Buchanan, E. (2013). The semantic priming project. Behavior Research Methods, 45(4), 1099-1114. https://doi.org/10.3758/s13428-012-0304-z

James, W. (2001). Psychology: The Briefer Course. Dover Publications.

https://books.google.it/books?id=eLmyPMGyKZUC

Johns, B. T., \& Jones, M. N. (2012). Perceptual inference through global lexical similarity. Topics in Cognitive Science, 4(1), 103-120. https://doi.org/10.1111/j.1756-8765.2011.01176.x

Jones, M. N., Hills, T. T., \& Todd, P. M. (2015). Hidden processes in structural representations: A reply to Abbott, Austerweil, and Griffiths (2015). https://doi.org/10.1037/a0039248

Jones, M. N., Kintsch, W., \& Mewhort, D. J. K. (2006). Highdimensional semantic space accounts of priming. Journal of Memory and Language, 55(4), 534-552. https://doi.org/10.1016/j.jml.2006.07.003

Jones, M. N., \& Mewhort, D. J. K. (2007). Representing word meaning and order information in a composite holographic lexicon. Psychological Review, 114(1), 1. https://doi.org/10.1037/0033-295X.114.1.1

Keuleers, E., \& Balota, D. A. (2015). Megastudies, crowdsourcing, and large datasets in psycholinguistics: An overview of recent developments. Taylor \& https://doi.org/10.1080/17470218.2015.1051065

Keuleers, E., \& Marelli, M. (2020). Resources for mental lexicon research: A delicate ecosystem. In V. Pirrelli, I. Plag, \& W. U. Dressler (Eds.), Word Knowledge and Word Usage: a Crossdisciplinary Guide to the Mental Lexicon. De Gruyter. https://doi.org/10.1515/9783110440577-005

Kintsch, W. (1988). The Role of Knowledge in Discourse Comprehension: A Construction-Integration Model. 
Psychological Review, 95(2), 163-182. https://doi.org/10.1037/0033-295X.95.2.163

Kintsch, W. (2000). Metaphor comprehension: A computational theory. Psychonomic Bulletin and Review, 7(2), 257-266. https://doi.org/10.3758/BF03212981

Kintsch, W. (2001). Predication. Cognitive Science, 25(2), 173202. https://doi.org/10.1207/s15516709 $\operatorname{cog} 2502 \_1$

Krizhevsky, A., Sutskever, I., \& Hinton, G. E. (2012). Imagenet classification with deep convolutional neural networks. Advances in Neural Information Processing Systems, 10971105. https://doi.org/10.1145/3065386

Lachmair, M., Dudschig, C., De Filippis, M., de la Vega, I., \& Kaup, B. (2011). Root versus roof: automatic activation of location information during word processing. Psychonomic Bulletin \& Review, 18(6), 1180-1188. https://doi.org/10.3758/s13423011-0158-x

Landauer, T. K., \& Dumais, S. T. (1997). A solution to Plato's problem: The latent semantic analysis theory of acquisition, induction, and representation of knowledge. Psychological Review, 104(2), 211. https://doi.org/10.1037/0033295x.104.2.211

Lazaridou, A., Marelli, M., \& Baroni, M. (2017). Multimodal word meaning induction from minimal exposure to natural text. Cognitive Science, 41, 677-705 https://doi.org/10.1111/cogs.12481

Lebois, L. A. M., Wilson-Mendenhall, C. D., \& Barsalou, L. W. (2015). Are Automatic Conceptual Cores the Gold Standard of Semantic Processing? The Context-Dependence of Spatial Meaning in Grounded Congruency Effects. Cognitive Science, 39(8), 1764-1801. https://doi.org/10.1111/cogs.12174

LeCun, Y., Bengio, Y., \& Hinton, G. (2015). Deep learning. Nature, 521, 436-444. https://doi.org/10.1038/nature14539

Lenci, A. (2008). Distributional semantics in linguistic and cognitive research. Italian Journal of Linguistics, 20(1), 1-31.

Louwerse, M. M. (2007). Symbolic or embodied representations: A case for symbol interdependency. Handbook of Latent Semantic Analysis, 107, 120

Louwerse, M. M. (2011). Symbol interdependency in symbolic and embodied cognition. Topics in Cognitive Science, 3(2), 273302. https://doi.org/10.1111/j.1756-8765.2010.01106.x

Lucas, M. (2000). Semantic priming without association: A metaanalytic review. Psychonomic Bulletin \& Review, 7(4), 618630. https://doi.org/10.3758/bf03212999

Mahon, B. Z. (2015a). The burden of embodied cognition. Canadian Journal of Experimental Psychology/Revue Canadienne de Psychologie Expérimentale, 69(2), 172-178. https://doi.org/10.1037/cep0000060

Mahon, B. Z. (2015b). What is embodied about cognition?
Language, Cognition and Neuroscience, 30(4), 420-429. https://doi.org/10.1080/23273798.2014.987791

Mahon, B. Z., \& Caramazza, A. (2005). The orchestration of the sensory-motor systems: Clues from neuropsychology. Cognitive Neuropsychology, 22(3-4), 480-494. https://doi.org/10.1080/02643290442000446

Mahon, B. Z., \& Caramazza, A. (2008). A critical look at the embodied cognition hypothesis and a new proposal for grounding conceptual content. Journal of Physiology-Paris, 102(1-3), 59-70. https://doi.org/10.1016/j.jphysparis.2008.03.004

Mandera, P., Keuleers, E., \& Brysbaert, M. (2017). Explaining human performance in psycholinguistic tasks with models of semantic similarity based on prediction and counting: A review and empirical validation. Journal of Memory and Language, 92, 57-78. https://doi.org/10.1016/j.jml.2016.04.001

Meyer, D. E., \& Schvaneveldt, R. W. (1971). Facilitation in recognizing pairs of words: evidence of a dependence between retrieval operations. Journal of Experimental Psychology, 90(2), 227. https://doi.org/10.1037/h0031564

Mikolov, T., Chen, K., Corrado, G., \& Dean, J. (2013). Efficient estimation of word representations in vector space. ArXiv Preprint ArXiv:1301.3781.

Miller, G. A. (1998). Nouns in wordnet. WordNet: An Electronic Lexical Database, 23-46.

Ostarek, M., \& Huettig, F. (2017). A task-dependent causal role for low-level visual processes in spoken word comprehension. Journal of Experimental Psychology: Learning Memory and Cognition, 43(8), 1215-1224. https://doi.org/10.1037/xlm0000375

Ostarek, M., \& Huettig, F. (2019). Six Challenges for Embodimen Research. Current Directions in Psychological Science, 096372141986644. https://doi.org/10.1177/0963721419866441

Pecher, D., Zeelenberg, R., \& Raaijmakers, J. (1998). Does pizza prime coin? Perceptual priming in lexical decision and pronunciation. Journal of Memory and Language, 38(4), 401418. https://doi.org/10.1006/jmla.1997.2557

Pezzulo, G., Barsalou, L. W., Cangelosi, A., Fischer, M. H., McRae, K., \& Spivey, M. (2013). Computational grounded cognition: a new alliance between grounded cognition and computationa modeling. Frontiers in Psychology, 3, 612. https://doi.org/10.3389/fpsyg.2012.00612

Pezzulo, G., Barsalou, L. W., Cangelosi, A., Fischer, M. H., Spivey, M., \& McRae, K. (2011). The mechanics of embodiment: A dialog on embodiment and computational modeling. Frontiers in Psychology, 2, 5. https://doi.org/10.3389/fpsyg.2011.00005 Pulvermüller, F. (2018). Neural reuse of action perception circuits 
for language, concepts and communication. Progress in Neurobiology, $\quad 160, \quad 1-44$. https://doi.org/10.1016/j.pneurobio.2017.07.001

Rescorla, R. A., \& Wagner, A. R. (1972). A Theory of Pavlovian Conditioning: Variations in the Effectiveness of Reinforcement and Nonreinforcement. Current Research and Theory, 64-99.

Schreuder, R., D’Arcais, G. B. F., \& Glazenborg, G. (1984). Effects of perceptual and conceptual similarity in semantic priming. Psychological Research, 45(4), 339-354. https://doi.org/10.1007/BF00309710

Seidenberg, M. S., \& McClelland, J. L. (1989). A Distributed, Developmental Model of Word Recognition and Naming. Psychological Review, 96(4), 523-568. https://doi.org/10.1037/0033-295X.96.4.523

Shelton, J. R., \& Martin, R. C. (1992). How semantic is automatic semantic priming? Journal of Experimental Psychology: Learning, Memory, and Cognition, 18(6), 1191. https://doi.org/10.1037/0278-7393.18.6.1191

Simmons, S., \& Estes, Z. (2008). Individual differences in the perception of similarity and difference. Cognition, 108(3), 781795. https://doi.org/10.1016/j.cognition.2008.07.003

Simonyan, K., \& Zisserman, A. (2014). Very deep convolutional networks for large-scale image recognition. ArXiv Preprint ArXiv:1409.1556.

Smith, D. A., Pezzelle, S., Franzon, F., Zanini, C., \& Bernardi, R. (2017). Can you see the (linguistic) difference? Exploring mass/count distinction in vision. IWCS 2017-12th International Conference on Computational Semantics-Short Papers.

Strozyk, J. V., Dudschig, C., \& Kaup, B. (2019). Do I need to have my hands free to understand hand-related language? Investigating the functional relevance of experiential simulations. Psychological Research, 83(3), 406-418. https://doi.org/10.1007/s00426-017-0900-8

Suárez, L., Tan, S. H., Yap, M. J., \& Goh, W. D. (2011). Observing neighborhood effects without neighbors. Psychonomic Bulletin and Review, 18(3), 605-611. https://doi.org/10.3758/s13423011-0078-9

Thornton, T., Loetscher, T., Yates, M. J., \& Nicholls, M. E. R. (2012). The Highs and Lows of the Interaction Between Word Meaning and Space. https://doi.org/10.1037/a0030467

Trumpp, N. M., Kliese, D., Hoenig, K., Haarmeier, T., \& Kiefer, M. (2013). Losing the sound of concepts: Damage to auditory association cortex impairs the processing of sound-related concepts. $\quad$ Cortex, $49(2), \quad 474-486$. https://doi.org/10.1016/j.cortex.2012.02.002

Tversky, A. (1977). Features of similarity. Psychological Review, 84(4), 327. https://doi.org/10.1037/0033-295X.84.4.327
Vedaldi, A., \& Lenc, K. (2015). Matconvnet: Convolutional neural networks for matlab. Proceedings of the 23rd ACM International Conference on Multimedia, 689-692. https://doi.org/10.1145/2733373.2807412

Wagenmakers, E. J., \& Farrell, S. (2004). AIC model selection using Akaike weights. In Psychonomic Bulletin and Review (Vol. 11, Issue 1, pp. 192-196). Psychonomic Society Inc. https://doi.org/10.3758/BF03206482

Westbury, C. (2016). Pay no attention to that man behind the curtain. The Mental Lexicon, 11(3), 350-374. https://doi.org/10.1075/ml.11.3.02wes

Wingfield, C., \& Connell, L. (2019). Understanding the role of linguistic distributional knowledge in cognition. https://doi.org/10.31234/osf.io/hpm4z

Yarkoni, T., Balota, D., \& Yap, M. (2008). Moving beyond Coltheart's N: A new measure of orthographic similarity. Psychonomic Bulletin and Review, 15(5), 971-979. https://doi.org/10.3758/PBR.15.5.971

Yee, E., Ahmed, S. Z., \& Thompson-Schill, S. L. (2012). Colorless green ideas (can) prime furiously. Psychological Science, 23(4), 364-369. https://doi.org/10.1177/0956797611430691

Zeiler, M. D., \& Fergus, R. (2014). Visualizing and understanding convolutional networks. European Conference on Computer Vision, 818-833. https://doi.org/10.1007/978-3-319-10590$1 \_53$

Zhang, R., Isola, P., Efros, A. A., Shechtman, E., \& Wang, O. (2018). The unreasonable effectiveness of deep features as a perceptual metric. Proceedings of the IEEE Conference on Computer Vision and Pattern Recognition, 586-595. https://doi.org/10.1109/CVPR.2018.00068

Zwaan, R. A. (2014). Embodiment and language comprehension: Reframing the discussion. Trends in Cognitive Sciences, 18(5), 229-234. https://doi.org/10.1016/j.tics.2014.02.008

Zwaan, R. A., \& Madden, C. J. (2005). Embodied sentence comprehension. Grounding Cognition: The Role of Perception and Action in Memory, Language, and Thinking, 224-245. https://doi.org/10.1017/CBO9780511499968.010

Zwaan, R. A., Stanfield, R. A., \& Yaxley, R. H. (2002). Language comprehenders mentally represent the shapes of objects. Psychological Science, 13(2), 168-171. https://doi.org/10.1111/1467-9280.00430 